\title{
Genome-wide identification, expression analysis and evolutionary relationships of the IQ67-domain gene family in common wheat (Triticum aestivum L.) and its progenitors
}

\section{Qinglin Ke}

Jiangxi Agricultural University

Huifan Sun

Jiangxi Agricultural University

Ruihan Luo

Jiangxi Agricultural University

Yan Zeng

Jiangxi Agricultural University

Mengxing Wang

Jiangxi Agricultural University

Yihan Li

Jiangxi Agricultural University

Zhimin Li

Jiangxi Agricultural University

Licao Cui ( $\square$ cuilicao@jxau.edu.cn )

Jiangxi Agricultural University

\section{Research Article}

Keywords: wheat, IQD gene family, expression profiles, polyploidization, asymmetric evolution.

Posted Date: January 27th, 2022

DOI: https://doi.org/10.21203/rs.3.rs-1234496/v1

License: (9) This work is licensed under a Creative Commons Attribution 4.0 International License.

Read Full License

Version of Record: A version of this preprint was published at BMC Genomics on April 5th, 2022. See the published version at https://doi.org/10.1186/s12864-022-08520-w. 


\section{Abstract}

\section{Background}

The plant-specific IQ67-domain (IQD) gene family plays an important role in plant development and stress responses. However, little is known about the IQD family in common wheat (Triticum aestivum $\mathrm{L}$ ), an agriculturally important crop that provides more than $20 \%$ of the calories and protein consumed in the modern human diet.

\section{Results}

We identified 125 IQDs in the wheat genome and divided them into four subgroups by phylogenetic analysis. The IQDs belonging to the same subgroup had similar exon-intron structure and conserved motif composition. Polyploidization contributed significantly to the expansion of IQD genes in wheat. Characterization of the expression profile of these genes revealed that a few T. aestivum (Ta)IQDs are tissue-specific, but most are ubiquitously expressed. The stress-induced expression pattern also revealed a potential role of TaIQDs in environmental adaptation, as TaIQD-2A-2, TaIQD-3A-9 and TaIQD-1A-7 were significantly induced by cold, drought and heat stresses, and could be candidates for functional validation and further used for molecular breeding. In addition, IQD genes in the A, B and D subgenomes displayed an asymmetric evolutionary pattern, as evidenced by their different gain or loss of member genes, expression levels and nucleotide diversity.

\section{Conclusions}

This study elucidated the biological functions and evolutionary relationships of the IQD gene family in wheat and revealed the divergent fates of IQD genes during polyploidization.

\section{Background}

As an intracellular second messenger, calcium $\left(\mathrm{Ca}^{2+}\right)$ is involved in plant growth and development as well as the regulation of abiotic and biotic stress responses [1]. The $\mathrm{Ca}^{2+}$ ion levels, induced by dosedependent intracellular signals transduced through $\mathrm{Ca}^{2+}$ sensors, differ in their spatiotemporal properties associated with the strength and duration of environmental challenges [2]. There are four major categories of $\mathrm{Ca}^{2+}$ sensor proteins in plants, namely calmodulins (CaMs), CaM-like proteins (CMLs), calcineurin $\mathrm{B}$-like proteins (CBLs), and $\mathrm{Ca}^{2+}$-dependent protein kinases (CDPKs) [3-5]. Upon sensing $\mathrm{Ca}^{2+}$, CaMs, CMLs and CBLs undergo conformational changes in their structures and interact with their target proteins to induce $\mathrm{Ca}^{2+}$ signals, while CDPKs contain an inherent kinase domain that can directly transduce the signal to the target protein when sensing $\mathrm{Ca}^{2+}$ signal [6]. CaMs are among the most common $\mathrm{Ca}^{2+}$ sensor proteins. Although lacking the catalytic activity, CaMs can interact and activate a wide spectrum of target proteins, and thereby play a crucial role in mediating physiological functions through their downstream target proteins. These target proteins include chaperones, metabolic enzymes, 
transcription factors, and kinases referred to as calmodulin-binding proteins (CaMBPs) [7]. CaMBPs are characterized by their calmodulin-binding domain (CaMBD), which consists of three conserved motifs, specifically, one $\mathrm{Ca}^{2+}$-independent motif (IQ motif), and two $\mathrm{Ca}^{2+}$-dependent motifs (I-5-10 motif and I-814 motif) [8]. The IQD proteins are common representatives of CaMBPs, characterized by a central region of 67 conserved amino acid residues, commonly known as IQ67 domain (IQD) protein families [9, 10]. The IQ67 domain consists of 1-3 copies of the IQ motif (IQxxxRGxxxR or [ILV]QxxxRxxxx[R, K]), 1-4 copies of the 1-5-10 motif ([FILVW]xxx[FILV]xxxx[FILVW]) and 1-4 copies of the 1-8-14 motif ([FILVW] xxxxxx[FAILVW] xxxxx[FILVW]) [11]. These features allow the IQ67 domain to form a basic amphiphilic helix structure, further endowing these proteins with specific roles [11].

In recent years, the research on the IQD gene family has attracted considerable attention in various model and non-model plants, such as Arabidopsis, Oryza sativa [11], Solanum lycopersicum [12], Brachypodium distachyon [13], Glycine max [14], Populus trichocarpa [15], Phyllostachys edulis [16], Zea mays [17], Cucumis sativus [18], Brassica rapa ssp. pekinensis [19], Vitis vinifera [20] and Solanum tuberosum [21]). Numerous studies have shown that the IQD genes are widely involved in various biological processes. For instance, AtIQD1 can promote glucosinolate accumulation to reduce insect herbivory in Arabidopsis [10, 22]. The microtubule-associated protein AtIQD5 controls cortical microtubule dynamics that promotes proper microtubule organization, and subsequent cell growth, shape formation and pavement cell shape morphogenesis [23, 24]. More recently, Arabidopsis thaliana IQDs were found to function as cellular scaffolds that facilitate preprophase band formation and cortical division zone establishment during symmetric cell division [25]. IQD12/ SUN regulates tomato shape by redistribution of fruit mass, and also plays important roles in the growth of floral organ and leaf morphology [26, 27]. SUN24 positively regulates seed germination by repressing the expression of two key ABA signaling genes (SIAB/3 and SIABI5) of the ABA signaling pathway in tomato [28]. In Gossypium hirsutum, knockdown of GhIQD31 and GhIQD32 negatively affected the responses of upland cotton to drought, salt and cold stresses [29]. $P d I Q D 10$ regulates second cell wall biosynthesis and impacts biomass formation in Populus [30]. Overexpression of BrIQD5 conferred drought stress tolerance to Chinese cabbage, possibly by interacting with CaMs and other drought-related proteins [19].

As one of the most successful crops since the dawn of agriculture, common wheat (Triticum aestivum L.) has expanded its original habitat from a limited area within the Fertile Crescent to a wide range of environments worldwide, making it the most widely grown and consumed crop [31]. Common wheat $(2 \mathrm{n}=$ $6 x=42, A A B B D D)$ is an allohexaploid species derived from two rounds of hybridization between three distinct diploid species, and is an informative system for analyzing the asymmetric evolutionary patterns between different subgenomes [32]. It originated from two natural interspecific hybridization events within the genera Triticum and Aegilops, which had similar but distinct genome structure and gene content that diverged between 2.5 and 6 million years ago [33]. First, Triticum urartu $(2 n=2 x=14, A A)$ hybridized with an uncertain grass that was highly similar to Aegilops speltoides $(2 n=2 x=14, S S)$ to generate the tetraploid species of wild emmer or Triticum turgidum $(2 n=4 x=28, A A B B)$ at about 0.5 to 3 million years ago. The subsequent polyploidization combined the genomes of Triticum turgidum and 
Aegilops tauschii $((2 n=2 x=14, D D)$ to form the allohexaploid genome of Triticum aestivum at around 8,000 years ago [34].

The completion of the genome sequence of hexaploid wheat has provided an opportunity to investigate gene families at the genome-wide level [35]. A systematic analysis of the IQD gene family during polyploidization is expected to accelerate molecular breeding in common wheat. In this study, 125 TaIQD genes were identified from the wheat genome. Their phylogenetic relationships, conserved motif composition, intron-exon structure and physicochemical characteristics of their proteins were comprehensively analyzed. In addition, we evaluated the expression pattern of TaIQDs during the stage of post anthesis and embryo development, and in response to various stresses, in which the proteins encoded by some TaIQD genes might potentially play a crucial role in stress resistance. The evolutionary relationships with its progenitors were systematically assessed. Comparative genomic analysis of TaIQD genes in wheat and its progenitors revealed asymmetric evolution and expansion during wheat polyploidization, as evidenced by their biased gene gain and loss, homoeologous gene expression and nucleotide diversity. This study can serve as a useful reference for unravelling the evolution of TaIQD genes and will further greatly contribute to breeding improvement in wheat.

\section{Results}

\section{Identification of IQD Genes in Wheat}

BLAST search [17], HMMER search [15], or both [21] were used to identify IQD genes in different species. A multifarious approach using the HMMER and BLAST programs as well as various online databases was used in our study. The study identified a total of 125 IQD genes in the wheat genome (Table S1). Since there is no standard nomenclature for IQD genes in wheat, the wheat IQD genes were designated as TaIQD-1A/1B/1D-1 to TaIQD-7A/7B/7D-3 for the A, $B$ and $D$ subgenomes according to their chromosomal location and homoeologous relationships, and TaIQD-U-1 to TaIQD-U-2 for unanchored genes. As shown in Table S1, the length of the TaIQD proteins ranged from 339 (TaIQD-1B-6) to 2,388 (TaIQD-3B-5) amino acids (aa) with an average of 785.46 aa, with corresponding molecular weight from 37.1 to $271.15 \mathrm{kDa}$, and isoelectric point from 4.93 (TalQD-2D-8) to 11.47 (TaIQD-4D-4). Noteworthy, all the IQD proteins has negative GRAVY values, indicating that these proteins have hydropathicity. The results of the subcellular localization revealed that 117 of the 125 (93.6\%) TaIQDs were only found in the nucleus, the remaining TaIQDs were found in chloroplast, mitochondrion, endoplasmic reticulum, cell membrane and cell wall.

\section{Sequence Alignment, Phylogenetic Analysis and Structure of TaIQDs}

The analysis of the domain conservation in the TaIQDs, after extracting the cores sequences of IQ motifs in wheat identified a total of 263 IQ motifs in wheat, with an average of 2.1 IQ motifs per protein, which was higher than that in rice (1.71), maize (1.65) and Arabidopsis (1.57). The length of the consecutive amino acid sequence of the IQ motifs ranged from 17 to 20 aa. As shown in Figure 1, amino acid residues Ile-6, Gln-7, Arg-11, Gly-12 and Arg-16 were determined to be conserved amino acids with the conservative ratio of more than $60 \%$. Notably, the conserved sequence ratio of Gln-7 was $100 \%$, 
suggesting that this amino acid may be essential for the biological function of IQD proteins. Moreover, a similar pattern was observed in Arabidopsis, rice and maize (Table S2) [11, 17]. Besides IQ motifs, the search for calmodulin-binding sites revealed that TaIQDs have one to five CaM-binding sites with the consecutive amino acid length $\geq 7$. Among them, TaIQD-2B-3 and TaIQD-2D-3 contain five CaM-binding sites, ranking as the most abundant CaM-binding domain containing TaIQDs. The predicted calmodulin interaction sites in 58 TaIQDs overlapped with the IQ motif (Table S3).

In order to assess the evolutionary relationships of wheat TaIQD genes, an unrooted phylogenetic tree was constructed based on the alignment of the full-length sequence of IQD proteins from wheat (125 proteins) and maize (26 proteins). The TaIQDs were classified into four subgroups designated as I, II, III and IV on the basis of the classification principle used in maize (Figure 2). The ratio of memberships within each subgroup in wheat was similar to that in maize, Arabidopsis and rice [11, 17]. Specifically, subgroup I had the most IQD proteins (68), followed by subgroup III (26) and subgroup IV (25), while subgroup II had the fewest with only six members (TaIQD-3A/3B/3D-4 and TaIQD-1A/1B/1D-7) (Table S4).

The exon-intron structure could also have certain reference value to understand the relative relationships of TaIQD genes. As shown in Figure 3, the number of exons of IQD genes ranged from 2 (TaIQD-5A-2) to 53 (TalQD-3B-5). The average exon length was $184.6 \mathrm{bp}$, whereas the intron length varied from 63 to $19,468 \mathrm{bp}$ with an average length of $356.18 \mathrm{bp}$, indicating that the noncoding regions were subjected to lower selection pressure, thereby exhibited higher sequence diversity. It is noteworthy that TalQDs grouped within the same subgroup shared a similar exon-intron structure and number of exons. However, the exonintron structure among different subgroups were significantly different. For example, subgroup I exhibited the most complex gene structure among all subgroups, suggesting that they may have versatile roles in plant growth and development.

Further analysis of the motif composition of TaIQD proteins predicted a total of 10 conserved motifs (Figure S1). The most abundant motif 1 and motif 7 were exclusively present in 75 and 46 TalQDs, respectively. Both motifs represented the core sequence region of the IQ motifs. Motif 1 represented the conventional IQ motif (IQxxxRGxxxxR), whereas motif 7 was relaxed version of the IQ motif ([IL]QxxxRxxxxR). Additionally, certain conserved motifs for specific subgroups were identified. For example, the subgroup I specific motif 5 and motif 9 were found to be exclusively located at the $\mathrm{N}$ terminal and C-terminal regions of TaIQD proteins, respectively. Motifs 2, 3, 4, 6 and 8 were uniquely present in Group I and Group IV. Subgroup II only contained motif 1, possibly due to its conserved exonintron structure. In addition, a conserved motif arrangement was observed within each subgroup, but different subgroups contained their specific organization, and we thus inferred that TaIQDs have conserved and diverse functions.

\section{Syntenic Relationships of TaIQD Genes in Wheat and Its Relatives}

The TaIQD genes were found to be unevenly distributed along the wheat chromosomes. Out of the 125 IQD genes identified in the wheat genome, a total of 123 TalQDs, comprising 41 for A, 42 for $B$ and 40 for D subgenomes, were mapped to the chromosomes (Figure S2). Most of the TaIQDs (81.6\%) had three 
copies associated with subgenomes A, B and D. Group 3 chromosomes contained more IQD genes than other chromosomes, with 11 IQD genes in the A, B and D subgenomes, respectively. In addition, group 6 chromosomes had the lowest number of $I Q D$ genes, with only two members for each subgenome. Most of the TaIQDs were located at the distal regions of the chromosome. Genetic and cytological studies have demonstrated that recombination events predominately occur at distal regions of the chromosome, but suppressed at pericentromeric regions [36, 37].

It should be noted that TaIQD-U-2 and TaIQD-U-1 were not located on definite chromosomes. Given that TaIQD-U-1 showed homology with TaIQD-7A-2 and TaIQD-7B-2, and TaIQD-U-2 was homologous with TaIQD-5A-2, we thus speculated that TaIQD-U-1 and TaIQD-U-2 were located at the middle of chromosome 7D and the top of chromosome 5D, respectively. The Pearson correlation coefficient between the number of TaIQDs and chromosome length was 0.23 with a p-value of 0.32 , which suggested that longer chromosomes do not necessarily contain more TalQD genes.

As the representative allopolyploid species, the genomic duplication of $A, B$ and $D$ subgenomes play an indispensable role in the expansion of the total gene dose within the genome. For this reason, we further performed the analysis of the syntenic relationships among different subgenomes. Ultimately, a total of 87 gene pairs consisting of 101 IQD genes were found to be syntenic genes. There were 25 homoeologous gene groups with the three complete copies associated with $\mathrm{A}, \mathrm{B}$ and $\mathrm{D}$. The TaIQD-5A/5B7, TaIQD-1B/1D-7and TalQD-1A/1D-3 syntenic groups were only observed for that between $A$ and $B, B$ and $D$, and $A$ and $D$, respectively. As an established software for evolutionary analysis of gene collinearity and synteny [38], the tandem repeat duplication events can also be identified by MCScanX. However, no tandem duplication was detected in our study, suggesting that genomic polyploidization led to the expansion of the IQD family in wheat. The Ka/Ks ratios for the $87 \mathrm{TaIQD}$ syntenic gene pairs were estimated and the values varied from 0.0234 to 0.5865 , with an average value of 0.1677 , suggesting that the IQD gene family experienced strong purifying selection pressure (Table S5). The divergence time of syntenic genes ranged from 0.88 to 32.90 million years ago (Mya) with the average of 9.55 Mya.

To further elucidate the evolutionary mechanism of $I Q D$ genes in wheat and its progenitors, a unified identification standard as described for wheat was used to identify the IQD genes in other species. A total of 232 IQD genes were identified, comprising 36 from Triticum urartu, 76 from Triticum dicoccoides, 78 from Triticum turgidum and 42 from Aegilops tauschii (Figure 4, Figure S3, Table S6). For the A subgenome, 36 IQD genes from Triticum turgidum showed syntenic relationships with those of wheat, followed by Triticum dicoccoides (32), and Triticum urartu (28). It was found that 20 TaIQDs of the A subgenome were also present in the three related species. We thus speculate that since these genes may have important biological functions, they have a definite conservation rate during evolution. In addition, seven IQD genes (TaIQD-1A-1, TaIQD-1A-2, TaIQD-2A-8, TaIQD-3A-5, TaIQD-3A-9, TaIQD-3A-10, TaIQD-5A-1) were identified as homologs between Triticum aestivum and Triticum dicoccoides as well as between Triticum aestivum and Triticum turgidum. However, no homologous genes were found between Triticum aestivum and Triticum urartu, suggesting that these homologous pairs might be formed after wheat tetraploidization. For the B subgenome, 36 IQDs were identified as syntenic gene pairs between Triticum 
aestivum and Triticum dicoccoides, and 37 between Triticum aestivum and Triticum turgidum. The TaIQD-3B-2 gene formed no homologous gene pairs or showed homologous relationships with other genes in other species. For the D subgenome, 40 TaIQDs showed homologous relationships with 39 IQD genes in Aegilops tauschii. Noteworthy, one IQD in Triticum aestivum and three IQDs in Aegilops tauschii showed no collinearity with the other species, suggesting that these genes might experience gene acquisition, gene loss or chromosome translocation after wheat polyploidization.

Furthermore, the calculation of the $\mathrm{Ka} / \mathrm{Ks}$ ratios revealed the orthologous relationships of IQD genes between wheat and its relatives (Table S7). The Ka/Ks ratios of 2, 10, 14 and 15 homologous gene pairs values were found to be higher than 1 in Triticum urartu, Aegilops tauschii, Triticum dicoccoides, Triticum turgidum, suggesting that these genes might undergo intense positive selection during the evolutionary process. In contrasty, the rest of the homologous gene pairs had negative $\mathrm{Ka} / \mathrm{Ks}$ ratios, suggesting that most of the IQD genes were subjected to purifying selection pressure.

\section{Expression Profiling of TalQD Genes in Various Stages}

The investigation of the potential biological functions of TaIQDs through the analysis of the expression profiles of TaIQDs in different developmental stages or tissues using publicly available RNA-seq data identified a greatly divergent expression pattern at ten time points after anthesis (Figure 5A). Among the $125 \mathrm{TalQD}$ genes, 50 genes were expressed at least in a specific sample with the FPKM $>1$. The expressed genes were divided into three major groups. The TaIQDs in the first group, such as TaIQD-2A-2, TaIQD-1D-3, TaIQD-2A-1, TaIQD-2A-4, TaIQD-2B-1, TaIQD-2D-1, TaIQD-3A-3, showed relatively high expression level at most time points, suggesting that these genes may play critical roles during the whole anthesis period in wheat. A total of 14 TaIQDs with a medium expression level were included in the second group. The remaining $16 \mathrm{TaIQDs}$, included in the third group, were almost not expressed at all the time points. In addition, we also found a number of time-point specific expressed genes. For example, TaIQD-2D-2 exhibited preferential expression at 17 DAA, while TaIQD-2B-2 was unbalanced expressed at 26 DAA time point.

Further analysis of the expression profiles at ten different time point during embryonal development (Figure 5B) showed that 64 TaIQD genes were expressed in at least one time point. Remarkably, no expression level was detected for 61 of the 125 (48.8\%) TalQD genes at the late leaf stage, suggesting

that TaIQD genes are not expressed during the process of cell aging. The tissue- and stage-specific IQDs were also identified. For instance, TaIQD-3A/3B/3D-2 showed expression bias in two cell types, preembryo and transition stages, implying that the three homoeologous genes may participate in early embryogenesis. In contrast, TaIQD-2A/2B/2D-1 showed relatively high expression in the late endosperm stage. TaIQD-3A/3B-4, TaIQD-4A/4B-1 and TaIQD-3D-4 were mainly expressed in seed coat. Thus, these results indicated that TaIQD genes were tissue-specific.

\section{Expression Profiling of TalQD Genes in Response to Various Stresses}


We also investigated the biological function of TaIQD genes in the response to various abiotic stresses, specifically cold, salt, drought/heat and metal starvation. The results showed that 45 genes expressed in response to cold stress (Figure 6A). The TaIQD-3D-10, TaIQD-3B-5, TaIQD-5A-7 and TaIQD-5B-9 genes were markedly upregulated. Remarkably, TaIQD-5A-7 showed about 6.62-fold higher expression level compared to the control. TaIQD-5B-9 was not expressed under untreated condition, but was markedly expressed in response to cold stress. Additionally, TaIQD-3D-3, TaIQD-5B-6, TaIQD-2B-4 and TaIQD-2A-5 were weakly expressed when subjected to cold treatment. Under salt stress (Figure 6B), seven genes showed upregulated expression patterns. In particular, TaIQD-5D-5 and TaIQD-1A-7 showed a 6.47 and 3.42-fold upregulation after exposure to salt. Moreover, the expression of TaIQD-2D-5 and TaIQD-5B-9 were induced in response to salt stress. When the plants were subjected to the combined stresses of drought and heat (Figure 6C) with the following six treatment and time point conditions (DS_1h, DS_6h, HS_1h, HS_6h, HD_1h and HD_6h), a total of 2, 2, 3, 7, 2 and 5 TalQD genes were upregulated and 2, 7, 13, 8, 10, and 7 TaIQD genes were downregulated, respectively. In particular, TaIQD-5A-6 showed 2.13-fold and 2.02-fold upregulation under the HS_6h and HD_6h treatments, and TaIQD-2A-5 showed more than 2-fold upregulation under the DS_1h, DS_6h, HS_6h and HD_6h treatments. The expression profiles of TaIQDs under phosphorus and iron deprivation were also determined (Figure 6D). Remarkably, the expression levels of TaIQD-4A-2, TaIQD-1B-4, TaIQD-5B-11, TaIQD-3D-3, TaIQD-7D-1 and TaIQD-U-1 showed more than 5 -fold upregulation than those of their respective control. The rest of the TaIQDs showed weak or moderate expression levels, suggesting that only a few genes are involved in the response to various stresses in wheat.

In order to gain a deep understanding into the expression of TaIQD family genes in response to multiple stresses, 9 TaIQD genes from four different subgroups were randomly selected to study their expression profiles under salt, drought, cold and heat stresses by Qrt-PCR analysis (Figure S4). Under salt stress, the selected TaIQDs were upregulated at different time points. For example, TaIQD-2A-2 and TaIQD-3A-9 were upregulated at all time points and reached their maximum expression level at $6 \mathrm{~h}$ and $1 \mathrm{~h}$, respectively. The expression of TaIQD-1A-7 peaked at $12 \mathrm{~h}$ with a 6.96-fold upregulation. At different time points of the cold stress treatment, two TaIQDs (TaIQD-2A-2 and TaIQD-5B-9) were upregulated, whereas TaIQD-3B-5 was downregulated at all time points. Meanwhile, some TaIQD genes showed variable expression profiles at different time points. For instance, TaIQD-5A-6 and TaIQD-3D-9 were downregulated at $6 \mathrm{~h}$, but upregulated at the remaining time points. In addition, the expression levels of the selected TaIQDs were analyzed after drought stress treatment. The expression levels of TaIQD-3B-5, TaIQD-3D-10 and TaIQD-3D9 were suppressed compared with those of the control. The expression of levels of TaIQD-1A-7 and TaIQD-3A-9 were significantly upregulated, and peaked at different times. Specifically, the expression of TaIQD-1A-7 peaked at $1 \mathrm{~h}$ and was upregulated 4.84-fold, whereas the expression of TaIQD-3A-9 was initially slightly upregulated and peaked at $12 \mathrm{~h}$. The results of the qRT-PCR analysis revealed that heat treatment had a marked effect on the expression patterns of TaIQDs. With the exception of TaIQD-3B-5 and TaIQD-5B-9, whose expression was inhibited compared with the control, the expression levels of a total of six TaIQDs (TaIQD-1A-7, 2A-2, 3A-9, 3B-11,3D-9 and 5A-6) peaked at $24 \mathrm{~h}$, suggesting that these TaIQDs might primarily function in the terminal stage in the response to heat injury. Notably, the 
expression of TaIQD-2A-2, TaIQD-3A-9 and TaIQD-1A-7 was significantly altered in response to salt, cold, heat and drought stresses, indicating that they might be excellent targets for the molecular breeding of wheat.

\section{Cis-regulatory Elements and Co-expression Network Analysis of TalQDs}

As the region containing the transcription factor binding site that initiates transcription, the promoter plays an essential role in controlling the expression of genes that are involved in plant organogenesis, hormone signal transduction and stress responses. To further elucidate the regulation mechanisms and potential functions of TaIQDs, the upstream $1.5 \mathrm{~kb}$ promoter region sequences from the start codon were obtained to characterize the cis-regulatory elements (Table S8 and Figure S5). In total, six hormonerelated cis-regulatory elements associated with gibberellin (GA), auxin, methyl jasmonate (MeJA), ethylene, salicylic acid (SA) and abscisic acid (ABA) were detected. Except for TaIQD-2B/2D-3, the majority of the TaIQDs had more than 13 hormone- or stress-responsive related cis-elements. In particular, gibberellin- (GARE-motif, P-box, TATC-box), MeJA- (CGTCA-motif, TGACG-motif), ABA- (ABRE), auxin(TGA-element, AuxRR-core), ethylene- (ERE) and salicylic acid- (SARE, TCA-element) were found in 59, $104,105,58,19$, and 32 TaIQDs, respectively. Abundant hormone-responsive cis-regulatory elements were enriched in the promoter regions of TaIQD-1B-4, TaIQD-5A-4, TaIQD-2B-4, TaIQD-3B-6, TaIQD-5A-8 and TaIQD-U-1. In addition, numerous abiotic stress cis-elements were also found, such as low-temperature responsive element LTR (53 genes), drought responsive element MBS (50 genes), salinity, osmolarity and heat responsive element W-box (57 genes), as well as dehydration responsive elements DRE (three genes), DRE core (64 genes) and DRE1 (17 genes). Additionally, three kinds of biotic stress related cisregulatory elements were also detected, including defense responsive TC-rich repeat elements (27 genes), wounding responsive element WUN-motif (23 genes) and wounding responsive element 3 (WRE3) (82 genes). These results implied that TaIQD genes might play critical roles in biotic and abiotic stresses, and might be involved in hormone stimulus responses.

MicroRNAs (miRNAs) can direct the cleavage of target mRNA or translation inhibition to regulate plant development and response to environmental fluctuations [39]. In this study, the putative miRNAs targeting the mNAs of TaIQDs were predicted by psRNATarget. A total of 20 miRNA-TaIQD putative targeting relationships comprising 13 miRNAs and 13 TaIQDs were predicted with more than $90 \%$ sequence alignment (Table S9). Specifically, tae-miR9653a precisely binds to TaIQD-1A-7 with $100 \%$ alignment. All the miRNAs silenced the post-transcriptional expression of TaIQDs through transcript cleavage. Moreover, except for miR1120c-TaIQD-2D-5, the rest of the miRNA-TaIQD interactions were found to act upstream of the IQ domains. Overall, these results suggest that miRNAs may have crucial roles in the posttranscriptional regulation of the expression of TaIQD, and further research on the miRNA-mediated interaction relationships will provide valuable information to understand the functional roles of TaIQDs in plant growth and development as well as stress responses.

To investigate the regulatory functions of TaIQDs associated with other wheat genes, the available 90 RNA-seq samples were used to construct a co-expression network (Figure 7A). The network consisted of a 
total of 1,708 nodes with eight TaIQDs and 67 other genes. Among them, the highly connected TaIQD-2B5 and TaIQD-6D-2, located at the core node position, were co-expressed with 44 (58.67\%) and 43 (57.33\%) related genes, respectively, suggesting that these two TaIQDs might play a central role in the regulatory network. Three TaIQD genes (TaIQD-2B-5, TaIQD-6B-2 and TaIQD-6D-2) had co-expressed relationships with CIPK1, PME31 and NADP-ME3. The genes co-expressed with TaIQDs were significantly enriched in functional categories that included phenylamine metabolism, metabolic pathways, phenylpropanoid biosynthesis, biosynthesis of secondary metabolites and other KEGG pathways (Figure 7B). GO enrichment analysis of the TaIQD co-expressed genes revealed that they were most enriched in the terms related to multiple developmental process (Figure 7C), such as L-phenylalanine catabolic process (GO:0006559), pollen development (GO:0009555), glucuronoxylan biosynthetic process (G0:0010417), as well as those related to response to various stresses, such as wounding (GO: G0:0009611), UV-B (GO:0010224), Karrikin (G0:0080167), jasmonic acid (GO:0009753), and oxidative stress (G0:0006979). Noteworthy, several stress response motifs (ARE, DRE, STRE, WRE3, GC-motif, W-box) and hormone regulators (ABRE, as-1, CGTCA-motif, P-box, TGACG-motif, TGA-element) were found in the promoter regions of these genes.

\section{Nucleotide Variation and Population Structure Analysis of TaIQD Genes}

The genetic landscapes with the genera Triticum and Aegilops have been comprehensively analyzed at the whole-genome level [31], but studies of the nucleotide variation patterns of TaIQDs are rather limited. By taking advantage of the cutting-edge analysis tools of whole-genome sequencing datasets, the nucleotide variation analysis uncovered 5,145 TaIQD-related SNPs, including 1,430, 1,297 and 2,418 for the A, B and D subgenomes, respectively. The majority of the SNPs were located within the upstream $(38.46 \%)$ or downstream (32.69\%) regions, followed by the intronic regions $(17.47 \%)$, while only $10.65 \%$ SNPs were in exonic regions (Table S10). Within the coding regions, we observed $4.30 \%$ synonymous and $2.93 \%$ non-synonymous SNPs with a synonymous versus non- synonymous ratio of 1.46 . Except for four members (TaIQD-1B-4, TaIQD-7A-2, TaIQD-U-7and TaIQD-U-2), 96.8\% of TaIQDs contained at least one variation site. Among them, TaIQD-5D-6 (282 SNPs), TaIQD-6A-2 (195 SNPs) and TaIQD-6D-2 (125 SNPs) were the most variable TaIQD genes.

The evolutionary relationships and population structure of the different subspecies were further studied at the sub-genomic level. For the A subgenome, PCA showed that the first principal component accounted for $61.4 \%$ of the total variance and mainly distinguished the Triticum urartu from the other species, whereas Triticum aestivum (landrace) was mainly distinguished by the second principal component (15.93\% of total variance), and Triticum turgidum was distinguished by the third (Figure 8B and 8C, Table S11). A more obvious subgroup that included from top to bottom Triticum urartu, Triticum dicoccoides, Triticum dicoccum, Triticum turgidum and Triticum aestivum, was identified through the phylogenetic tree (Figure 8A). Admixture analysis provided similar evidence (Figure 8D). When $\mathrm{K}=2$, the species Triticum urartu was firstly recognized. With the increase of $\mathrm{K}$ to 3 , the landraces and cultivars of common wheat were separated from the others. With the continuous increase of the $\mathrm{K}$ value, a certain proportion of gene flow between common wheat and its progenitors was observed, indicating the continuous gene flow 
between its diploid and tetraploid ancestors and hexaploid wheat during and after the process of polyploidization. The of nucleotide diversity increased gradually from the diploid wheat (Triticum urartu) to tetraploid wheat (Triticum dicoccoides, Triticum dicoccum and Triticum turgidum) and then to hexaploid wheat (Triticum aestivum). The genetic diversity of Triticum dicoccum and Triticum turgidum populations was basically the same, but a significant genetic loss ( $40.2 \%$ reduction) occurred in the Triticum dicoccoides population during domestication. The $F_{s t}$ is an important index used to evaluate gene flow intensity and population differentiation [40]. If the $F_{s t}$ value is larger than 0.25 , populations are considered to be extremely divergent [41]. HIn this study, the $F_{s t}$ values between Triticum urartu and other populations were larger than those within the other populations, which was consistent with the results of the phylogenetic relationships with the deviated cluster groups of the Triticum urartu population.

For the B subgenome, all accessions were assigned to five subgroups according to their biological sources. The first, second and third components mainly captured the difference of Triticum dicoccoides, Triticum dicoccum and Triticum turgidum, respectively. Within the phylogenetic tree, the Triticum dicoccum population is definitely separated from the others, but there is no obvious boundary between the landrace and modern cultivar accessions for both the tetraploid wheat and hexaploid wheat. The same population affinities were recovered in the stacked bar based on the Admixture analysis. When $\mathrm{K}=$ 2, a genetic admixture was observed for Triticum dicoccoides and Triticum turgidum. However, it was not until $\mathrm{K}$ increased to 4 that Triticum turgidum formed a relatively independent subgroup. When $\mathrm{K}$ increased above 5 , the landrace and hexaploid wheat gradually diverged, but there was still obvious genetic admixture between the two populations. We further evaluated the genetic diversity of the B subgenome for different populations. The nucleotide diversity of TaIQDs decreased continuously from Triticum dicoccoides (0.2472) to Triticum dicoccum (0.1685), and ultimately to Triticum turgidum wheat (0.1282). In contrast, the genetic diversity increased from landraces $(0.1483)$ to cultivar $(0.2067)$ of hexaploid wheat (Figure 9, Figure S6).

We also profiled the nucleotide variation atlas of TaIQDs for the D subgenome. As described in subparagraph A of Figure S7, a significant genetic divergence was observed between the D subgenome of hexaploid wheat and its ancestral species Aegilops tauschii. Identical results were obtained when the high $F_{s t}$ values were calculated for wheat varieties versus Aegilops tauschii $(0.602)$ and wheat landraces versus Aegilops tauschii (0.579), which suggested that these populations were highly differentiated between each other. Moreover, the average nucleotide diversity of Aegilops tauschii ranked the highest among the studied populations of different subgenomes. However, the nucleotide diversity of the $D$ subgenome decreased $\sim 85 \%$ from the ancestral species to hexaploid wheat. In summary, the evolutionary patterns of TaIQD genes provide novel insights into the process of wheat polyploidization, which might be useful in wheat genetic research and germplasm resource utilization in the future.

\section{Discussion}

\section{The First Characterization of IQD Gene Family in Wheat}


The plant-specific IQD gene family, encoding a class of calmodulin-binding proteins involved in calcium signaling pathways, plays essential roles in plants the coordination of a wide range of developmental processes and responses to environmental stimuli [11]. The IQD gene family is believed to have originated during the early evolution of land plants, possibly before the divergence of bryophyte and vascular plant lineages at around 450-700 Mya [42], but not later than the differentiation between gymnosperms and angiosperms about $300 \mathrm{Mya}$, as there was at least one putative IQD-like gene present in Physcomitrella patens and nine IQD genes in pine identified by EST and full-length cDNA sequences [43].

The identification and characterization of IQD family genes have been comprehensively performed at the genome-wide level in model and non-model plant species. Wheat is one of the most important cultivated grain crops worldwide, contributing approximately a fifth of the food consumption for the majority of the human populations [35]. With the advent of high-throughput sequencing technologies and novel computational algorithms over the past decades, the completion of the wheat genome assembly has made it easy to access sequence-level information for breeding programs.

In total, 125 non-redundant IQD genes were identified in the wheat genome. The number of IQD genes in wheat is more than three times higher than that in Arabidopsis thaliana (33) [11], Brachypodium distachyon (23) [13], Brassica rapa ssp. pekinensis (35) [19], maize (26) [17], Oryza stative (29) [11], Populus trichocarpa (40) [15], potato (23) [21], and tomato (34) [12]. Compared with its progenitors, the number of IQD in Triticum aestivum is approximately three times higher than in Triticum urartu (36) and Ae. tauschii (42), whereas the ratio of TalQDs between Triticum aestivum and Triticum dicoccoides or between Triticum aestivum and Triticum turgidum was approximately 1.5. This finding is consistent with those of previous studies on various gene families, including the TaPRX (374 in Triticum aestivum, 159 in Triticum urartu and 169 in Aegilops. tauschii) [44], TaPK (3,269 in Triticum aestivum, 1,213 in Triticum urartu and 1,448 in Aegilops tauschii) [45], WOX (43 in Triticum aestivum, 23 in Triticum dicoccoides, 28 in Triticum. turgidum, 16 in Triticum urartu and 13 in Aegilops tauschii) [46] and Hsp70 (113 in Triticum aestivum, 79 in Triticum dicoccoides and 30 in Aegilops tauschii) [47] gene families. This phenomenon can be explained by the two rounds of polyploidization that directly led to the expansion of the IQD genes in wheat.

Among all the identified TaIQDs, the analysis of the physicochemical characteristics showed a significant difference. For example, the TaIQD-3B-5 had only 339 amino acid residues and a pl value of 11, whereas the TaIQD-3B-5 had 2,388 amino acid residues and a lower pl value of 5.65. For amino acids, the $\mathrm{pl}$ is the average pKa values of the amine and carboxyl groups, which are equal to the $\mathrm{pH}$ value when the molecule is not charged [48]. The variation of physicochemical properties might lead to the diversity of protein function. Furthermore, we predicted the subcellular localization of TaIQDs. The results showed that $123(98.4 \%)$ wheat IQD proteins were found in the nucleus, which was a significantly higher proportion than that for the IQD protein family in Arabidopsis (48.5\%), rice (55.2\%) and Brachypodium distachyon $(69.6 \%)[11,13]$. 
A comparative genomic analysis of $I Q D$ gene family members from wheat and maize was performed to investigate the phylogenetic relationships. The phylogenetic analysis revealed that TaIQD proteins fell into four subgroups within the tree. Subgroup I was the largest subgroup, followed by subgroup III, which was in agreement with the results in maize, Arabidopsis and rice [11,17]. The TaIQD proteins in the same subclade tend to cluster with each other more than proteins from the same species in different subclades. Meanwhile, Genes within the same subgroup had a similar gene structure and conserved motif composition. Motif 1 and motif 7, which were the most common motifs, represented the conventional IQ67 motif (IQxxxRGxxxxR) and a more relax version ([IL]QxxxRxxxxR). In addition, the subgroup specific motifs were also identified, which may be related to the functional diversification of TaIQDS.

\section{The Potential Function of TaIQDs Might Be in Plant Growth, Development and Response to Various Stresses}

Cis-acting regulatory elements have been implicated in the spatiotemporal regulation of gene expression throughout the life of plants [49], and have been shown to be activated by certain transcription factors to regulate the expression of stress-related genes when plants are exposed to adverse conditions [50]. We identified a series of cis-acting elements related to light response, plant growth and development, hormone signal transduction, and stress response present widely in the promoter regions of most TaIQD genes. These cis-acting regulatory elements consistently appeared within the promoter regions of IQDs in various species $[20,21,51]$, indicating that IQD genes might plays a very important role in regulating plant growth, development and physiological processes. Since our results were obtained through predictive analysis, the potential regulation mechanism still needs to be confirmed by further experiments.

The biological functions of IQD proteins have been extensively studied in multiple plants, especially model plants [52]. The elucidation of expression patterns of TalQDs during plant growth and development will provide new insight into the potential functions of their proteins. TaIQD-1D-1 showed a relatively high expression level during the whole stage of anthesis. Its orthologous gene AtIQD1 encodes a protein found in microtubules that interacts with the KLCR1 (kinesin light chain related 1) protein to expedite the cellular transportation [10]. Our results suggest that TaIQD-1D-1 might be involved in mediating the transition from vegetative to reproductive development in barley. In Arabidopsis, AtIQD5 protein was reported to be involved in the process of cell shape morphogenesis, whereas its orthologous gene in wheat, TalQD-5B-1 showed preferential expression in embryonal leaf middle and mature stages [24], implying that TaIQD-5B1 might be involved in these processes.

As sensible organisms that are not able to move, terrestrial plants are often exposed to a wide array of adverse challenges, such as drought, high salinity, extreme temperatures and pathogen infection [53]. To adapt to such environmental stimuli in an appropriate manner, plants have evolved complex signal transduction pathways that enable them to perceive stress signal and coordinate their growth and development [54]. The IQD proteins have been reported to be induced by various stresses in plants. In Chinese cabbage, overexpression of BrIQD5 confers plants drought tolerance, while BrlQD5-silenced plants exhibited drought sensitivity [19]. Gossypium hirsutum plants with knockdown of GhIQD31 and 
GhIQD32 showed decreased drought and salt tolerance [29]. In this study, we identified several cold, salt, drought/heat and metal starvation induced IQD genes in wheat. Most TaIQDs tend to be induced by drought/heat rather than cold stress. Notably, TaIQD-2B-2, TaIQD-2D-3 and TaIQD-5A-10 were significantly upregulated by more than 20 -fold after exposure to drought/heat, suggesting their essential roles in the response to drought/heat stress. Although several of the identified candidate IQD genes could serve as targets for subsequent genetic isolation and functional investigation in wheat, further experiments are needed to determine the biological functions of TaIQDs.

As a systems biology approach for determining the potential interactions among genes, WGCNA is an effective method to identify clusters of highly correlated genes, summarizing clusters, relating modules to sample traits, and for calculating module membership [55]. The genes adjacent to TaIQDs were found to be related to signaling pathways, cellular process, metabolic process, reproductive process,

developmental process, and response to stimulus. Several genes in the co-expression network were found to have potential interaction with multiple TaIQD genes, such as CIPK1, PME31 and NADP-ME3. In Arabidopsis, the $\mathrm{Ca}^{2+}$ regulated protein kinase CIPK1 was reported to contribute to modulate phosphate starvation in plants [56]. The protein NADP-malic enzyme, encoded by the gene NADP-ME3, participates in pyruvate metabolism and carbon fixation by catalyzing the reaction of (S)-malate and NADP ${ }^{+}[57]$. Meanwhile, the protein encoded by PME31 can adjust cell wall component and structure to positively improve salt resistance [58]. Interestingly, the calmodulin genes CaM4 and WRKY71 were predicted to be co-expressed with TaIQD-2B-5 and TaIQD-6D-2. CaM4 can enhance salt tolerance and produce NO in Arabidopsis [59], and WRKY71 plays a crucial role in promoting plant flowering to complete its life cycle by conferring plant salt tolerance [60]. These results suggested that TaIQDs are widely involved in wheat growth, development and various stress responses. Moreover, several of the identified candidate IQD genes could serve as targets for subsequent genetic isolation and functional investigation in barley as well as in other cereal crops.

\section{The Asymmetric Evolution Patterns of IQD Genes in Hexaploid Wheat}

Plant polyploidization, together with the asymmetry in the process of co-evolution between different subgenomes, has contributed to sufficient genetic variation for environmental adaption [61]. A large number of studies have found that polyploid species have undergone asymmetric evolution in all aspects of their genomes. The draft genome of Brassica oleracea revealed the multi-layered asymmetrical evolution patterns between the Brassica subgenomes, such gene loss between subgenomes, amplification of tandem duplication and transposable elements, preferential enrichment for specific pathways and divergence in gene expression [61]. Asymmetric selection of defense-response genes also has led to ecotype change in Brassica napus [62]. More recent studies have provided t evidence indicating that common wheat and cotton might have experienced asymmetric selection between different subgenomes $[63,64]$.

In order to find the asymmetric evolution patterns of TaIQDs, the member composition of the IQD gene families between common wheat and its progenitors was compared. For the A subgenome, a total of 4 (2 
for $2 \mathrm{~A}$ and 2 for $7 \mathrm{~A}$ ) IQD genes were lost and 10 (three in $1 \mathrm{~A}$, two in $3 \mathrm{~A}$, two fin $4 \mathrm{~A}$ and three in $5 \mathrm{~A}$ ) were identified when compared with Triticum urartu. By contrast, only one in 1A and two in 3A IQDs were identified and one (in 2A) was lost after hexaploidization compared to Triticum turgidum. For the $B$ subgenome, three $I Q D$ genes belonging to $2 \mathrm{~B}, 3 \mathrm{~B}$ and $4 \mathrm{~B}$ were gained in hexaploid wheat. For the $\mathrm{D}$ subgenome, one more IQD gene on 1D was found in contrast to Aegilops tauschii. Noteworthy, the shorter the time taken by the ancestors to form the hexaploid wheat, the higher the consistency of the gene composition for IQD genes. In additions, previous studies have demonstrated that the gene number tend to be reversed towards diploid levels through gene loss following plant polyploidization [65]. In contrast, a slight increase was observed for IQD genes in wheat. We hypothesized that the exogenous introgression after the formation of hexaploid wheat may lead to the expansion of the TaIQD gene family, or any other complex mechanisms that remain unclear.

The presence of the homoeologous triads (composed of A, B and D genome copies) led us to examine the divergence from gene structure to biological function between subgenomes. The results indicated that around $\sim 52.94 \%$ of the homoeologous genes from the A, B and D subgenomes have different predicted exon numbers. However, there was only 1 out of 34 homoeologous gene pairs (TalQD$1 A / 1 B / 1 D-10$ ) that showed the divergent motif composition. Analysis of a total of 90 RNA-seq data revealed that most of the homoeologous genes showed a similar expression pattern. At the whole genome level, approximately $30 \%$ of the wheat homoeologous genes showed a biased expression pattern with lower or higher expression levels for a single homoeolog compared with the other two [32]. Regarding the IQD genes in wheat, a small portion of TaIQDs were differentially expressed in different stages/tissues or in response to exposure to stress. For example, TaIQD-2A-5 and TaIQD-2D-5 were upregulated in salt stress, TaIQD-2B-5 was downregulated in heat stress. Under cold treatment, TaIQD-2A5 was downregulated, and TaIQD-2B-5was upregulated, and TaIQD-2D-5 was not expressed. These findings suggest the potential sub-functionalization or neo-functionalization of these genes.

As the most common type of genomic variation, SNPs have become an increasingly powerful molecular genetic marker for producing high-resolution genetic maps, linkage disequilibrium analysis, and markerassisted breeding [66]. By taking advantage of the high-confident TaIQD-related SNPs, the nucleotide diversity was calculated for each subgenomes with $B>A>D$ in hexaploid wheat, which was basically consistent with previous studies [31,67]. The asymmetric patterns of IQD genes in wheat will broaden our understanding on wheat genome evolution and will support research into the various important crops in the Triticum genus.

\section{Conclusions}

This study comprehensively analyzed for the first time the wheat IQD genes. A total of 125 TaIQDs were thoroughly identified in the wheat genome. We also categorized the genes into four subgroups according to the phylogenetic relationships between wheat and maize, which was supported by the exon-intron structure and conserved motif composition analysis. The expression and co-expression analysis showed that the TaIQDs were widely involved in plant development, and in the response to environmental 
stresses. The expression of TaIQD-2B-5, TaIQD-6B-2 and TaIQD-6D-2 was significantly induced by exposure to various types of abiotic stresses, which might make these genes excellent targets for the molecular breeding of wheat. In addition, some of the IQD genes in the A, B and D subgenomes had different gene gain and loss rates, expression patterns and nucleotide diversity. Taken together, the findings of this study provide new insights into the biological function and molecular evolution of the IQD gene family in wheat during polyploidization.

\section{Materials And Methods}

\section{Identification of the IQD Gene Family in Wheat}

The sequence-related data of wheat were downloaded from the Ensembl Plants database (http://plants.ensembl.org/index.html). The previously reported IQD protein sequences from Arabidopsis thaliana, rice (Oryza sativa) and maize (Zea mays) were considered as reference sequences to blast against the proteins in the wheat whole genome using BLASTP with e-value $\leq 1 \mathrm{e}-5$ and identity $\geq 50 \%$. In addition, the hidden Markov model (HMM) profiles of the IQ domain (Pfam ID: PF00612) were retrieved from the Pfam database (http://pfam.xfam.org/) and used as query to search the wheat proteins using HMMER v3.0 (https://www.ebi.ac.uk/Tools/hmmer/). The redundant sequences from BLAST and HMMER were manually removed and further verified using the online Pfam (http://pfam.xfam.org/search/sequence) databases. Only candidate proteins that had the IQ domain were retained. To confirm the presence of all candidate genes, a BLASTN search was conducted against the wheat expressed sequence tag (ESTs) downloaded from the National Center for Biotechnology Information (NCBI, Bethesda, MD, USA) database (https://www.ncbi.nlm.nih.gov/) using the following criteria: e-value $\leq 1 \mathrm{e}-5$ and identity $\geq 80 \%$. The physicochemical characteristics of TaIQD proteins, including molecular weight (MW), theoretical isoelectric point ( $\mathrm{pl}$ ) and grand average of hydropathy (GRAVY), were calculated by the online ExPASy server (https://web.expasy.org/protparam/). The subcellular localization of TaIQDs was predicted by the predictor tool in the Plant-mPLoc server (http://www.csbio.sjtu.edu.cn/bioinf/plant-multi/). The calmodulin target database (http://calcium.uhnres.utoronto.ca/ctdb/ctdb/sequence.html) was used to identify the putative CaMbinding sites of TalQDs.

\section{Phylogenetic Analysis, Gene Structure and Conserved Motif Analysis of TaIQD Genes}

The full-length IQD proteins from wheat as well as those from maize were used to generate multiple sequence alignment by ClustalX v1.83 with default parameters. The WebLogo tool (http://weblogo.berkeley.edu/logo.cgi) was used to display the sequence logo of the IQ motif. Then, the phylogenetic tree was constructed by the neighbor-joining ( $N J)$ method using MEGAX v10.0 [68] with 1,000 replications, $95 \%$ partial deletion and a Poisson model. Ten motifs were scanned using MEME v.5.0.5 with a width ranging from 8 to 50 amino acids [69] (http://meme-suite.org/tools/meme). The Gene Structure Display Server [70] (http://gsds.cbi.pku.edu.cn/) was used to visualize the exon-intron composition of TalQD genes. 


\section{Gene Duplication, Homoeologous Relationships and Ka/Ks Estimation}

The chromosomal location of TaIQDs was obtained from the wheat genome annotation file (http://plants.ensembl.org/index.html) and diagrams were drawn using MG2C v2.1

(http://mg2c.iask.in/mg2c_v2.0/). In order to establish the syntenic relationships of IQD genes among the $A, B$ and D subgenomes in wheat, we performed an all $v s$. all BLASTP search for all the TaIQD proteins. TaIQD s clustered within the same branches of the phylogenetic tree and displaying more than 95\% similarity between each other were considered as homoeologous groups. According to their chromosome location, MCScanX was used to determine the syntenic relationships of the TaIQD gene family among the A, B and D subgenomes [38].

To evaluate the evolutionary relationships of IQD genes between wheat and its relatives (Triticum urartu, Triticum dicoccoides, Triticum turgidum and Aegilops tauschii), the proteins of these four species were retrieved from the Ensembl Plants database. Then, we used the same methods and criteria as described for wheat to identify the IQD genes in Triticum urartu, Triticum dicoccoides, Triticum turgidum and Aegilops tauschii. The synteny analysis of IQD genes between the homologs of wheat and its relatives was performed using MCScanX. The syntenic maps were visualized using Circos v0.67. The Ka (nonsynonymous substitution)/Ks (synonymous substitution) ratio value was calculated using the PAML package to estimate the divergence of the homologous genes [71].

\section{Analysis of Expression Profiles of TaIQD Genes Using Transcriptome Data}

A total of 90 spatiotemporal and stress treatment RNA-seq samples of wheat, including embryonal stage, 3 to 26 days after anthesis (DAA) leaves, and abiotic stresses (cold, heat, drought, salt, and P and iron starvation), were downloaded from the NCBI Sequence Read Archive (SRA) database. Detail sample information are listed in Table S12. The fragments per kilobase per million (FPKM) value was calculated using HISAT2 v2.1.0 and the StringTie v1.3.5 pipeline. The $\log _{2}(F P K M+1)$ value of TalQDs was used to generate the expression heatmaps using the pheatmap package in $\mathrm{R}$.

\section{Cis-acting Elements and Regulatory Network Analysis}

The $1.5 \mathrm{~kb}$ upstream DNA sequences from the gene transcription initiation site of TalQDs were extracted and submitted to the PlantCARE online database (http://bioinformatics.psb.ugent.be/webtools/plantcare/html/) to search for the putative cis-acting elements within the promoter region. The cDNA sequences of TaIQD genes were uploaded to the psRNATarget (http://plantgrn.noble.org/psRNATarget/) to find the candidate miRNA target sites. The coexpression network between TaIQDs and other related genes were constructed using the weighted gene co-expression network analysis (WGCNA) package in R according to their expression levels generated by the RNA-seq data. The co-expressed relationships with weighted values larger than 0.25 were retained for subsequent analysis. The Gene Ontology (GO) and Kyoto Encyclopedia of Genes and Genomes (KEGG) enrichment analyses of the co-expressed genes were performed using the KOBAS software 
(http://bioinfo.org/kobas), the potential relevancy between TaIQDs and other wheat genes was visualized using Cytoscape v3.8.0.

\section{Plant Material, Stress Treatment, RNA Extraction and qRT-PCR Analysis}

Seeds of Triticum aestivum landrace 'Chinese Spring' were geminated on petri dishes under dark condition, and cultured in the growth chamber with a $16 \mathrm{~h}$ light/ $8 \mathrm{~h}$ dark cycle at $23 \pm 1^{\circ} \mathrm{C}$. The three-leaf stage seedlings were subjected to abiotic treatments. For salt, drought, cold and heat stresses, the plants were subjected to treatment with $150 \mathrm{mM} \mathrm{NaCl}, 20 \%$ PEG-6000, $4^{\circ} \mathrm{C}$ and $42^{\circ} \mathrm{C}$ conditions, respectively. The leaves were sampled at 0, 1, 6, 12 and $24 \mathrm{~h}$ with three independent biological replications, and the untreated plants were used as controls. The sampled leaves were rapidly pre-cooled in liquid nitrogen and then stored at $-80^{\circ} \mathrm{C}$ for subsequent analysis.

The plant RNA Kit Reagent (Omega Bio-Tek Inc., Norcross, GA, USA) was used to isolate total RNA. The cDNA was synthesized using the Evo M-MLV RT Mix Kit (Accurate Biology, Changsha, China). The quantitative real-time polymerase chain reaction (qRT-PCR) analysis was performed using a SYBR® Green Premix Pro Taq HS qPCR Kit (Accurate Biology, Changsha, China) on an Applied Biosystems ${ }^{\text {TM }} 7500$ Real-Time PCR System (Thermo Fisher Scientific Inc., Waltham, MA, USA). The Primer Premier v5.0 software was used to design the primer sequences for TaIQD genes. The Elongation Factor 1-Alpha gene was used as the internal control. The primer information is listed in Table S13. The PCR cycling conditions were as follows: $95^{\circ} \mathrm{C}$ for $30 \mathrm{~s}$ for 1 cycle, followed by 40 cycles of $95^{\circ} \mathrm{C}$ for $5 \mathrm{~s}, 60^{\circ} \mathrm{C}$ for $30 \mathrm{~s}$. The $2^{-\triangle \Delta C T}$ method was used to evaluate the $m$ mRNA relative expression levels of TaIQDs [72]. The significance analysis was performed using the t-test package in R. The 0.05 and 0.01 significance level are indicated by one asterisk $(*)$ and two asterisks $(* \star)$, respectively.

\section{Nucleotide Variation and Population Structure Analysis}

The early released whole-genome sequencing population from the genera Triticum and Aegilops were used to identify the nucleotide variation of TaIQD genes [31]. The genomic variation data was downloaded from the SnpHub (http://wheat.cau.edu.cn/WheatUnion/b_4/). The following criteria were used for filtration: with the minor allele frequency (MAF) $>0.05$ and $<0.95$, the maximum missing rate $<$ 0.1. To avoid the difference due to sampling, the same number of samples for different population was selected as much as possible. Therefore, we use a total of 27 Triticum urartu, 28 Triticum dicoccoides, 26 Triticum dicoccum, 10 Triticum turgidum, 26 Triticum aestivum (landrace), 20 Triticum aestivum (cultivar) and 30 Aegilops tauschii (Table S14). The principal component analysis (PCA) was performed using the smartpca subroutine in EIGENSOFT v6.1.4 [73]. An unrooted phylogenetic tree was generated by the neighbor-joining method with the parameters (1,000 bootstrap replications, $95 \%$ partial deletion and Poisson model), using MEGA X software. The population structure was analyzed by Admixture 1.3 [74] with the following parameters: the number of subgroups $\mathrm{K}$ ranged from 2 to 10, 10,000 times iteration and each $\mathrm{K}$ value was repeated five times. The VCFtools v0.1.16 genome toolbox was used to calculate 
the nucleotide diversity $(\pi)$ and fixation index $\left(F_{s t}\right)$. The above-mentioned analysis was performed for the $A, B$ and $D$ subgenomes, respectively.

\section{Abbreviations}

aa: amino acids

ABA: abscisic acid

CaM: calmodulin

CML: CaM-like

CBL: calcineurin B-like

CDPK: $\mathrm{Ca}^{2+}$-dependent protein kinase

CaMBP: calmodulin-binding protein

CaMBD: calmodulin-binding domain

DAA: days after anthesis

EST: expressed sequence tag

FPKM: fragments per kilobase per million

$F_{s t}$ fixation index

GA: gibberellin

GO: Gene Ontology

GRAVY: grand average of hydropathy

HMM: Hidden Markov Model

IQD: IQ67-domain

Ka: non-synonymous substitution

Ks: synonymous substitution

MAF: minor allele frequency

MeJA: methyl jasmonate 
MW: molecular weight

NJ: neighbor-joining

PCA: principal components analysis

pl: theoretical isoelectric point

qRT-PCR: quantitative real time polymerase chain reaction

SA: salicylic acid

SNP: single nucleotide polymorphism

SRA: Sequence Read Archive

WRE3: wounding responsive element 3

$\pi$ : nucleotide diversity

\section{Declarations}

\section{Ethics approval and consent to participate}

The common wheat (Triticum aestivum L.) cultivar Chinese Spring was grown and collected by College of Bioscience and Engineering, Jiangxi Agricultural University (Nanchang, China), and all samples from this cultivar was adopted for all experiment. These plant materials don't include any wild species at risk of extinction. No specific permits are required for sample collection in this study. We comply with relevant institutional, national, and international guidelines and legislation for plant study.

\section{Consent for publication}

Not applicable.

\section{Availability of data and materials}

All data supporting the conclusions of this article are provided within the article and its additional files. The sequences of Arabidopsis thaliana, Oryza sativa, Triticum urartu, Triticum dicoccoides, Triticum turgidum, Aegilops tauschii and Triticum aestivum are available in the Ensemble Plants database (http://plants.ensembl.org/index.html). The gene expression data was downloaded from the NCBI database (http://www.ncbi.nlm.nih.gov/geo/) under accession number PRJNA497810, PRJNA532455, PRJNA257938, PRJNA529036, PRJNA253535 and PRJNA487922. The genomic variation data was downloaded from the SnpHub (http://wheat.cau.edu.cn/WheatUnion/b_4/).

\section{Competing interests}


The authors declare that the research was conducted in the absence of any commercial or financial relationships that could be construed as a potential conflict of interest.

\section{Funding}

This research was mainly funded by the National Natural Science Foundation of China (Grant No. 32060458), Jiangxi Natural Science Foundation (Grant No. 20202BAB215002), and partially supported by the Science and Technology Research Project of Jiangxi Provincial Department of Education (Grant No. GJJ180241). The funders had no role in study design, data collection and analysis, decision to publish, or preparation of the manuscript.

\section{Authors' contributions}

Y.L. and L.C. designed the study. Q.K. and H.S. performed the data analysis. R.L., Y.Z. and M.W. collected the plant materials and performed the qRT-PCR experiments. Q.K. and R. L. validated the data. Q.K., Z.L., M.W. and L.C. wrote the manuscript. All authors read and approved the final manuscript.

\section{Acknowledgements}

We thank the High-Performance Computing center of Northwest A\&F University for providing computational resources in this work.

\section{References}

1. Dodd, A.N., Kudla, J., Sanders, D.: The language of calcium signaling. Annu Rev Plant Biol. 61, 593620 (2010).

2. Aldon, D., Mbengue, M., Mazars, C., Galaud, J.P.: Calcium Signalling in Plant Biotic Interactions. Int J Mol Sci. 19, (2018).

3. Ranty, B., Aldon, D., Galaud, J.P.: Plant calmodulins and calmodulin-related proteins: multifaceted relays to decode calcium signals. Plant Signal Behav. 1, 96-104 (2006).

4. Hrabak, E.M., Chan, C.W., Gribskov, M., Harper, J.F., Choi, J.H., Halford, N., Kudla, J., Luan, S., Nimmo, H.G., Sussman, M.R. et al. The Arabidopsis CDPK-SnRK superfamily of protein kinases. Plant Physiol. 132, 666-680 (2003).

5. Luan, S., Kudla, J., Rodriguez-Concepcion, M., Yalovsky, S., Gruissem, W.: Calmodulins and calcineurin B-like proteins: calcium sensors for specific signal response coupling in plants. Plant Cell. 14 Suppl, S389-400 (2002).

6. Zuo, R., Hu, R., Chai, G., Xu, M., Qi, G., Kong, Y., Zhou, G.: Genome-wide identification, classification, and expression analysis of CDPK and its closely related gene families in poplar (Populus trichocarpa). Mol Biol Rep. 40, 2645-2662 (2013). 
7. Kudla, J., Becker, D., Grill, E., Hedrich, R., Hippler, M., Kummer, U., Parniske, M.: Advances and current challenges in calcium signaling. New Phytol. 218, 414-431 (2018).

8. Rhoads, A.R., Friedberg, F.: Sequence motifs for calmodulin recognition. Faseb j. 11, 331-340 (1997).

9. Abel, S., Bürstenbinder, K., Müller, J.: The emerging function of IQD proteins as scaffolds in cellular signaling and trafficking. Plant Signal Behav. 8, e24369 (2013).

10. Bürstenbinder, K., Möller, B.: The IQD Family of Calmodulin-Binding Proteins Links Calcium Signaling to Microtubules, Membrane Subdomains, and the Nucleus. Plant Physiol. 173, 1692-1708 (2017).

11. Abel, S., Savchenko, T., Levy, M.: Genome-wide comparative analysis of the IQD gene families in Arabidopsis thaliana and Oryza sativa. BMC Evol Biol. 5, 72 (2005).

12. Huang, Z., Van Houten, J., Gonzalez, G., Xiao, H., van der Knaap, E.: Genome-wide identification, phylogeny and expression analysis of SUN, OFP and YABBY gene family in tomato. Mol Genet Genomics. 288, 111-129 (2013).

13. Filiz, E., Tombuloglu, H., Ozyigit, I.I.: Genome wide analysis of IQ67 domain (IQD) gene families in Brachypodium distachyon. Plant Omics. 6, 425-432 (2013).

14. Feng, L., Chen, Z., Ma, H., Chen, X., Li, Y., Wang, Y., Xiang, Y.: The IQD gene family in soybean: structure, phylogeny, evolution and expression. PLoS One. 9, e110896 (2014).

15. Ma, H., Feng, L., Chen, Z., Chen, X., Zhao, H., Xiang, Y.: Genome-wide identification and expression analysis of the IQD gene family in Populus trichocarpa. Plant Sci. 229, 96-110 (2014).

16. Wu, M., Li, Y., Chen, D., Liu, H., Zhu, D., Xiang, Y.: Genome-wide identification and expression analysis of the IQD gene family in moso bamboo (Phyllostachys edulis). Sci Rep. 6, 24520 (2016).

17. Cai, R., Zhang, C., Zhao, Y., Zhu, K., Wang, Y., Jiang, H., Xiang, Y., Cheng, B.: Genome-wide analysis of the IQD gene family in maize. Mol Genet Genomics. 291, 543-558 (2016).

18. Ge, Q., Wang, X., Li, H., Ren, Z., Wang, L.: Genome-wide Identification and Analysis of IQD/SUN Gene Family in Cucumber. Genomics and Applied Biology (2019).

19. Yuan, J., Liu, T., Yu, Z., Li, Y., Ren, H., Hou, X., Li, Y.: Genome-wide analysis of the Chinese cabbage IQD gene family and the response of BrIQD5 in drought resistance. Plant Mol Biol. 99, 603-620 (2019).

20. Liu, Z., Haider, M.S.: Comprehensive Sequence Analysis of IQD Gene Family and their Expression Profiling in Grapevine (Vitis vinifera). Genes. 11, (2020).

21. Mei, C., Liu, Y., Dong, X., Song, Q., Wang, H., Shi, H., Feng, R.: Genome-Wide Identification and Characterization of the Potato IQD Family During Development and Stress. Front Genet. 12, 693936 (2021).

22. Levy, M., Wang, Q., Kaspi, R., Parrella, M.P., Abel, S.: Arabidopsis IQD1, a novel calmodulin-binding nuclear protein, stimulates glucosinolate accumulation and plant defense. Plant J. 43, 79-96 (2005).

23. Liang, H., Zhang, Y., Martinez, P., Rasmussen, C.G., Xu, T., Yang, Z.: The Microtubule-Associated Protein IQ67 DOMAIN5 Modulates Microtubule Dynamics and Pavement Cell Shape. Plant Physiol. 177, 1555-1568 (2018). 
24. Mitra, D., Klemm, S., Kumari, P., Quegwer, J., Möller, B., Poeschl, Y., Pflug, P., Stamm, G., Abel, S., Bürstenbinder, K.: Microtubule-associated protein IQ67 DOMAIN5 regulates morphogenesis of leaf pavement cells in Arabidopsis thaliana. J Exp Bot. 70, 529-543 (2019).

25. Kumari, P., Dahiya, P., Livanos, P., Zergiebel, L., Kölling, M., Poeschl, Y., Stamm, G., Hermann, A., Abel, S., Müller, S. et al. IQ67 DOMAIN proteins facilitate preprophase band formation and division-plane orientation. Nat Plants. 7, 739-747 (2021).

26. Wu, S., Xiao, H., Cabrera, A., Meulia, T., van der Knaap, E.: SUN regulates vegetative and reproductive organ shape by changing cell division patterns. Plant Physiol. 157, 1175-1186 (2011).

27. Xiao, H., Jiang, N., Schaffner, E., Stockinger, E.J., van der Knaap, E.: A retrotransposon-mediated gene duplication underlies morphological variation of tomato fruit. Science. 319, 1527-1530 (2008).

28. Bi, L., Weng, L., Jiang, Z., Xiao, H.: The tomato IQD gene SUN24 regulates seed germination through ABA signaling pathway. Planta. 248, 919-931 (2018).

29. Yang, X., Kirungu, J.N., Magwanga, R.O., Xu, Y., Pu, L., Zhou, Z., Hou, Y., Cai, X., Wang, K., Liu, F.: Knockdown of GhIQD31 and GhIQD32 increases drought and salt stress sensitivity in Gossypium hirsutum. Plant Physiol Biochem. 144, 166-177 (2019).

30. Badmi, R., Payyavula, R.S., Bali, G., Guo, H.B., Jawdy, S.S., Gunter, L.E., Yang, X., Winkeler, K.A., Collins, C., Rottmann, W.H. et al. A New Calmodulin-Binding Protein Expresses in the Context of Secondary Cell Wall Biosynthesis and Impacts Biomass Properties in Populus. Front Plant Sci. 9, 1669 (2018).

31. Zhou, Y., Zhao, X., Li, Y., Xu, J., Bi, A., Kang, L., Xu, D., Chen, H., Wang, Y., Wang, Y.G. et al. Triticum population sequencing provides insights into wheat adaptation. Nat Genet. 52, 1412-1422 (2020).

32. Ramírez-González, R.H., Borrill, P., Lang, D., Harrington, S.A., Brinton, J., Venturini, L., Davey, M., Jacobs, J., van Ex, F., Pasha, A. et al. The transcriptional landscape of polyploid wheat. Science. 361, (2018).

33. Walkowiak, S., Gao, L., Monat, C., Haberer, G., Kassa, M.T., Brinton, J., Ramirez-Gonzalez, R.H., Kolodziej, M.C., Delorean, E., Thambugala, D. et al. Multiple wheat genomes reveal global variation in modern breeding. Nature. 588, 277-283 (2020).

34. Berkman, P.J., Visendi, P., Lee, H.C., Stiller, J., Manoli, S., Lorenc, M.T., Lai, K., Batley, J., Fleury, D., Simková, H. et al. Dispersion and domestication shaped the genome of bread wheat. Plant Biotechnol J. 11, 564-571 (2013).

35. Appels, R., Eversole, K., Feuillet, C., Keller, B., Rogers, J., Stein, N., Pozniak, C.J., Stein, N., Choulet, F., Distelfeld, A. et al. Shifting the limits in wheat research and breeding using a fully annotated reference genome. Science. 361, (2018).

36. Sandhu, D., Gill, K.S.: Structural and functional organization of the '1S0.8 gene-rich region' in the Triticeae. Plant Mol Biol. 48, 791-804 (2002).

37. Künzel, G., Korzun, L., Meister, A.: Cytologically integrated physical restriction fragment length polymorphism maps for the barley genome based on translocation breakpoints. Genetics. 154, 397$412(2000)$. 
38. Wang, Y., Tang, H., Debarry, J.D., Tan, X., Li, J., Wang, X., Lee, T.H., Jin, H., Marler, B., Guo, H. et al. MCScanX: a toolkit for detection and evolutionary analysis of gene synteny and collinearity. Nucleic Acids Res. 40, e49 (2012).

39. Song, X., Li, Y., Cao, X., Qi, Y.: MicroRNAs and Their Regulatory Roles in Plant-Environment Interactions. Annu Rev Plant Biol. 70, 489- 525 (2019).

40. Wright, S.: The genetical structure of populations. Ann Eugen. 15, 323-354 (1951).

41. Fong, M.Y., Rashdi, S.A., Yusof, R., Lau, Y.L.: Genetic Diversity, Natural Selection and Haplotype Grouping of Plasmodium knowlesi Gamma Protein Region II (PkyRII): Comparison with the Duffy Binding Protein (PkDBPaRII). PLoS One. 11, e0155627 (2016).

42. Hedges, S.B.: The origin and evolution of model organisms. Nat Rev Genet. 3, 838-849 (2002).

43. Bowe, L.M., Coat, G., dePamphilis, C.W.: Phylogeny of seed plants based on all three genomic compartments: extant gymnosperms are monophyletic and Gnetales' closest relatives are conifers. Proc Natl Acad Sci U S A. 97, 4092-4097 (2000).

44. Yan, J., Su, P., Li, W., Xiao, G., Zhao, Y., Ma, X., Wang, H., Nevo, E., Kong, L.: Genome-wide and evolutionary analysis of the class III peroxidase gene family in wheat and Aegilops tauschii reveals that some members are involved in stress responses. BMC Genomics. 20, 666 (2019).

45. Yan, J., Su, P., Wei, Z., Nevo, E., Kong, L.: Genome-wide identification, classification, evolutionary analysis and gene expression patterns of the protein kinase gene family in wheat and Aegilops tauschii. Plant Mol Biol. 95, 227-242 (2017).

46. Shi, L., Wang, K., Du, L., Song, Y., Li, H., Ye, X.: Genome-Wide Identification and Expression Profiling Analysis of WOX Family Protein-Encoded Genes in Triticeae Species. Int J Mol Sci. 22, (2021).

47. Lai, D.L., Yan, J., Fan, Y., Li, Y., Ruan, J.J., Wang, J.Z., Fan, Y., Cheng, X.B., Cheng, J.P.: Genome-wide identification and phylogenetic relationships of the Hsp70 gene family of Aegilops tauschii, wild emmer wheat (Triticum dicoccoides) and bread wheat (Triticum aestivum). 3 Biotech. 11, 301 (2021).

48. Moldoveanu, S.C., David, V.: Properties of Analytes and Matrices Determining HPLC Selection. Selection of the HPLC Method in Chemical Analysis 189-230 (2017).

49. Yu, S., Sun, Q., Wu, J., Zhao, P., Sun, Y., Guo, Z.: Genome-Wide Identification and Characterization of Short-Chain Dehydrogenase/Reductase (SDR) Gene Family in Medicago truncatula. Int J Mol Sci. 22, (2021).

50. Hadiarto, T., Tran, L.S.: Progress studies of drought-responsive genes in rice. Plant Cell Rep. 30, 297310 (2011).

51. Rehman, A., Peng, Z., Li, H., Qin, G., Jia, Y., Pan, Z., He, S., Qayyum, A., Du, X.: Genome wide analysis of IQD gene family in diploid and tetraploid species of cotton (Gossypium spp.). Int J Biol Macromol. 184, 1035-1061 (2021).

52. Guo, C., Zhou, J., Li, D.: New Insights Into Functions of IQ67-Domain Proteins. Front Plant Sci. 11, 614851 (2020).

53. Zhu, J.K.: Abiotic Stress Signaling and Responses in Plants. Cell. 167, 313-324 (2016). 
54. Verma, V., Ravindran, P., Kumar, P.P.: Plant hormone-mediated regulation of stress responses. BMC Plant Biol. 16, 86 (2016).

55. Langfelder, P., Horvath, S.: WGCNA: an R package for weighted correlation network analysis. BMC Bioinformatics. 9, 559 (2008).

56. Lu, X., Li, X., Xie, D., Jiang, C., Wang, C., Li, L., Zhang, Y., Tian, H., Gao, H.: The Ca(2+) -regulated protein kinase CIPK1 integrates plant responses to phosphate deficiency in Arabidopsis thaliana. Plant Biol (Stuttg). 22, 753-760 (2020).

57. Wheeler, M.C., Tronconi, M.A., Drincovich, M.F., Andreo, C.S., Flügge, U.I., Maurino, V.G.: A comprehensive analysis of the NADP-malic enzyme gene family of Arabidopsis. Plant Physiol. 139, 39-51 (2005).

58. Yan, J., He, H., Fang, L., Zhang, A.: Pectin methylesterase31 positively regulates salt stress tolerance in Arabidopsis. Biochem Biophys Res Commun. 496, 497-501 (2018).

59. Zhou, S., Jia, L., Chu, H., Wu, D., Peng, X., Liu, X., Zhang, J., Zhao, J., Chen, K., Zhao, L.: Arabidopsis CaM1 and CaM4 Promote Nitric Oxide Production and Salt Resistance by Inhibiting SNitrosoglutathione Reductase via Direct Binding. PLoS Genet. 12, e1006255 (2016).

60. Yu, Y., Wang, L., Chen, J., Liu, Z., Park, C.M., Xiang, F.: WRKY71 Acts Antagonistically Against SaltDelayed Flowering in Arabidopsis thaliana. Plant Cell Physiol. 59, 414-422 (2018).

61. Liu, S., Liu, Y., Yang, X., Tong, C., Edwards, D., Parkin, I.A., Zhao, M., Ma, J., Yu, J., Huang, S. et al. The Brassica oleracea genome reveals the asymmetrical evolution of polyploid genomes. Nat Commun. 5, 3930 (2014).

62. Lu, K., Wei, L., Li, X., Wang, Y., Wu, J., Liu, M., Zhang, C., Chen, Z., Xiao, Z., Jian, H. et al. Whole-genome resequencing reveals Brassica napus origin and genetic loci involved in its improvement. Nat Commun. 10, 1154 (2019).

63. Hao, C., Jiao, C., Hou, J., Li, T., Liu, H., Wang, Y., Zheng, J., Liu, H., Bi, Z., Xu, F. et al. Resequencing of 145 Landmark Cultivars Reveals Asymmetric Sub-genome Selection and Strong Founder Genotype Effects on Wheat Breeding in China. Mol Plant. 13, 1733-1751 (2020).

64. Wang, M., Tu, L., Lin, M., Lin, Z., Wang, P., Yang, Q.: Asymmetric subgenome selection and cisregulatory divergence during cotton domestication. Nat Genet. 49, 579-587 (2017).

65. Sankoff, D., Zheng, C., Zhu, Q.: The collapse of gene complement following whole genome duplication. BMC Genomics. 11, 313 (2010).

66. Lai, K., Duran, C., Berkman, P.J., Lorenc, M.T., Stiller, J., Manoli, S., Hayden, M.J., Forrest, K.L., Fleury, D., Baumann, U. et al. Single nucleotide polymorphism discovery from wheat next-generation sequence data. Plant Biotechnol J. 10, 743-749 (2012).

67. Cheng, H., Liu, J., Wen, J., Nie, X., Xu, L., Chen, N., Li, Z., Wang, Q., Zheng, Z., Li, M. et al. Frequent intraand inter-species introgression shapes the landscape of genetic variation in bread wheat. Genome Biol. 20, 136 (2019).

68. Kumar, S., Stecher, G., Li, M., Knyaz, C., Tamura, K.: MEGA X: Molecular Evolutionary Genetics Analysis across Computing Platforms. Mol Biol Evol. 35, 1547-1549 (2018). 
69. Bailey, T.L., Boden, M., Buske, F.A., Frith, M., Grant, C.E., Clementi, L., Ren, J., Li, W.W., Noble, W.S.: MEME SUITE: tools for motif discovery and searching. Nucleic Acids Res. 37, W202-208 (2009).

70. Hu, B., Jin, J., Guo, A.Y., Zhang, H., Luo, J., Gao, G.: GSDS 2.0: an upgraded gene feature visualization server. Bioinformatics. 31, 1296-1297 (2015).

71. Yang, Z.: PAML 4: phylogenetic analysis by maximum likelihood. Mol Biol Evol. 24, 1586-1591 (2007).

72. Livak, K.J., Schmittgen, T.D.: Analysis of relative gene expression data using real-time quantitative PCR and the 2(-Delta Delta C(T)) Method. Methods. 25, 402-408 (2001).

73. Price, A.L., Patterson, N.J., Plenge, R.M., Weinblatt, M.E., Shadick, N.A., Reich, D.: Principal components analysis corrects for stratification in genome-wide association studies. Nat Genet. $\mathbf{3 8}$, 904-909 (2006).

74. Alexander, D.H., Novembre, J., Lange, K.: Fast model-based estimation of ancestry in unrelated individuals. Genome Res. 19, 1655-1664 (2009).

\section{Figures}


A
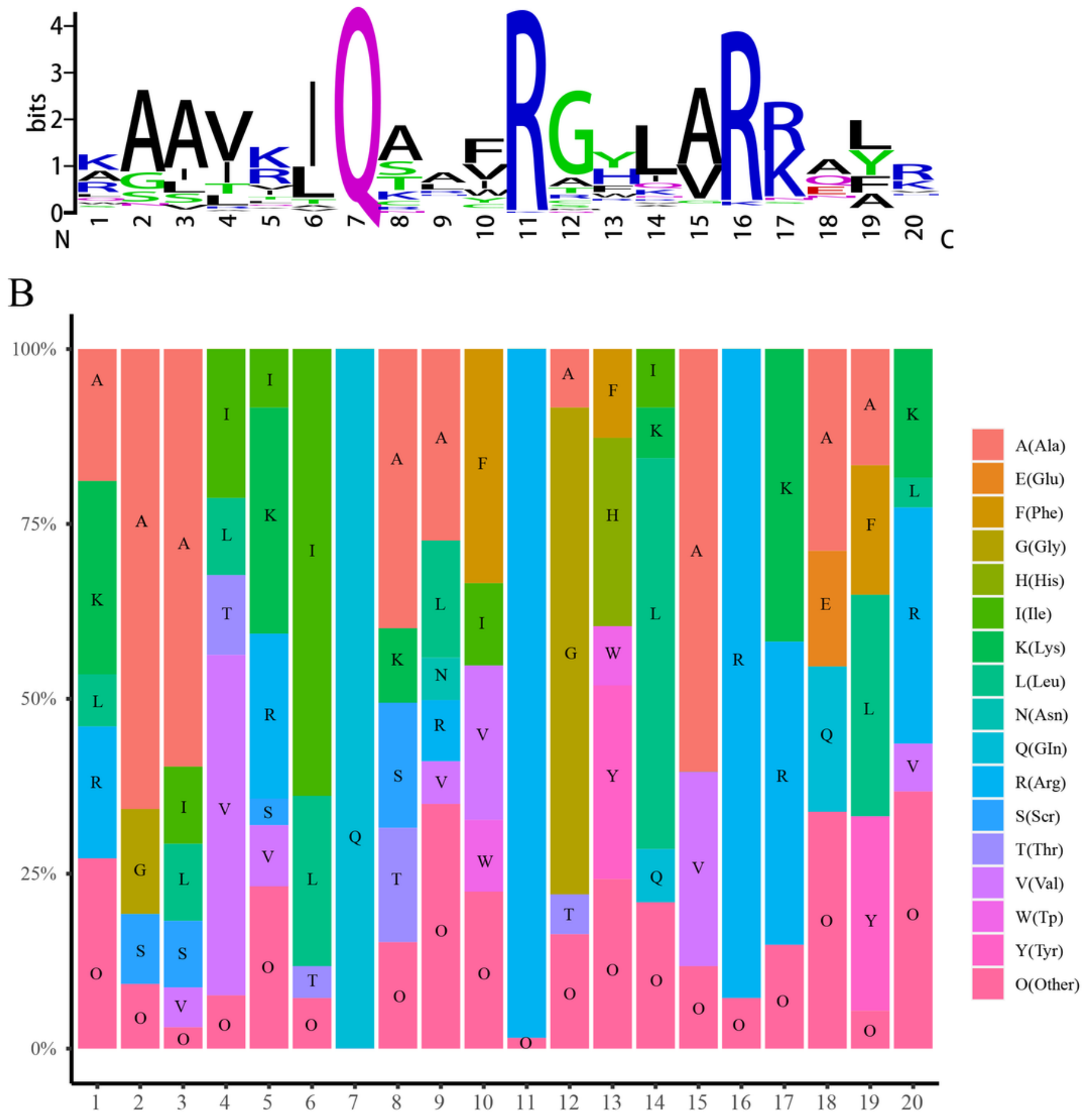

Figure 1

Conserved residue analysis of IQ domains. The height of each residue indicates the conservation rate. A: Sequence logo generated by WebLogo, B: Amino acid composition. 


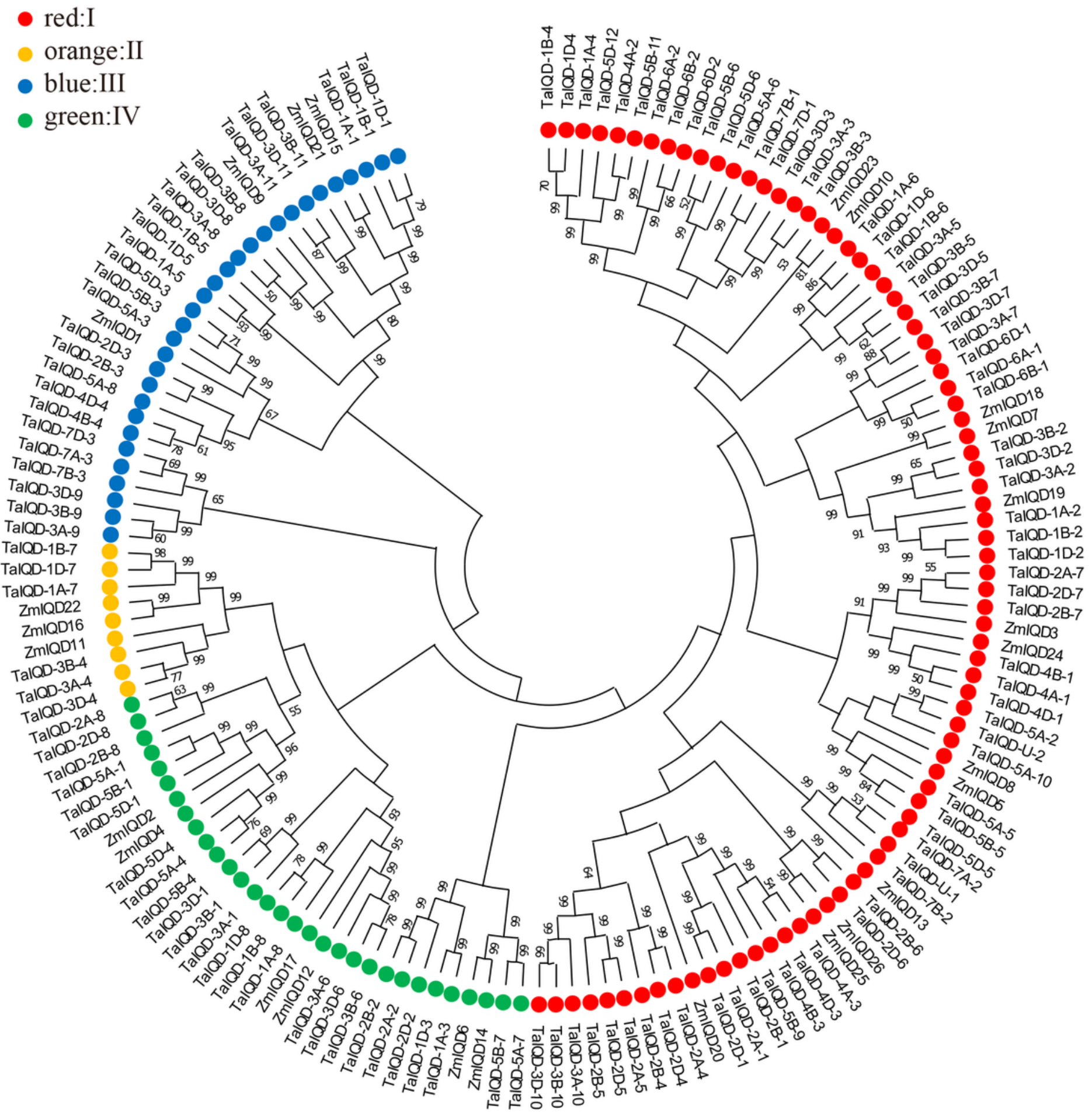

Figure 2

Phylogenetic tree analysis of IQD proteins from wheat and maize. The four IQD subgroups were marked with different colors. Red for subgroup I, orange for subgroup II, blue for subgroup III, green for subgroup IV. 

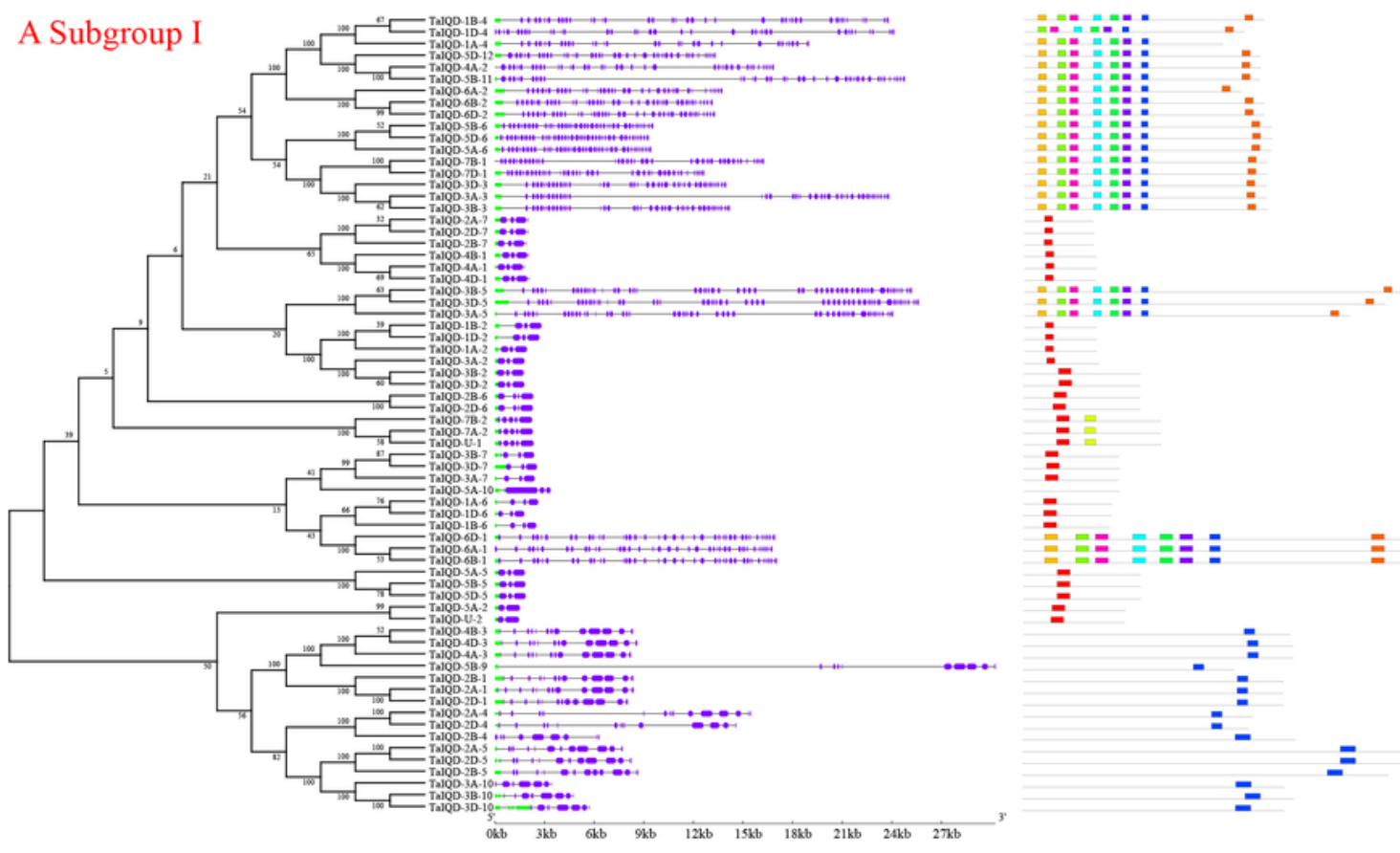

B Subgroup II

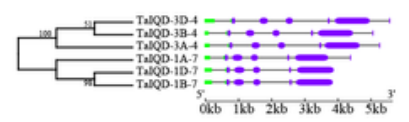

$\equiv$

C Subgroup III
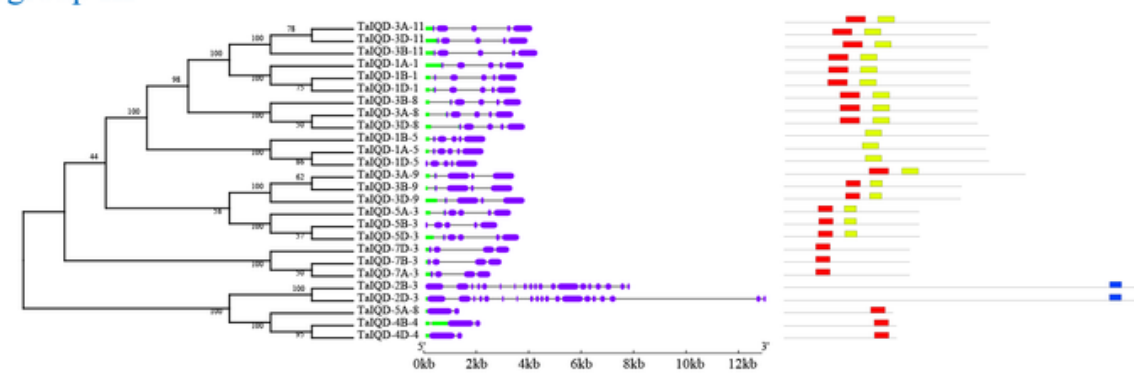

D Subgroup IV
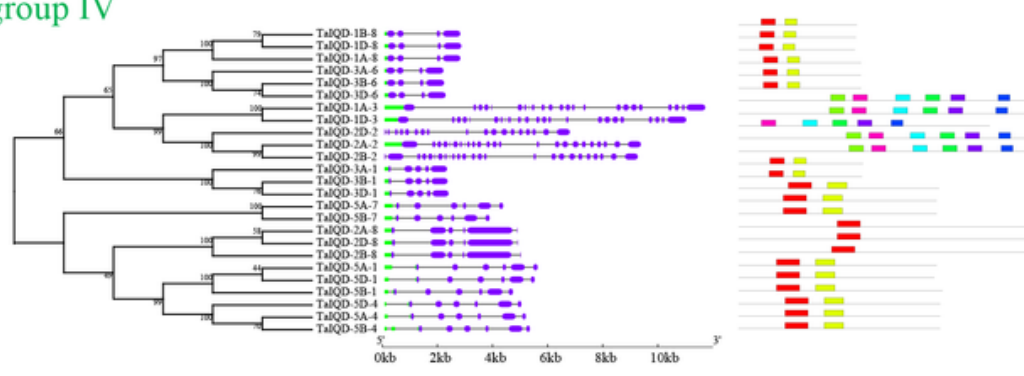

- Exon - Intron - five_prime_UTR - three_prime_UTR

motif $1=$ motif $2=\operatorname{motif} 3 \square$ motif $4 \square$ motif 5

motif $6 \square$ motif $7 \square$ motif $8 \square$ motif $9 \square$ motif 10

\section{Figure 3}

Phylogenetic relationships, exon-intron organization and motif composition of TalQDs. Exons and introns are indicted by grep rectangles and black lines. Conserved motifs are represented by colored boxed. A: Subgroup I, B: Subgroup II, C: Subgroup III, D: Subgroup IV. 


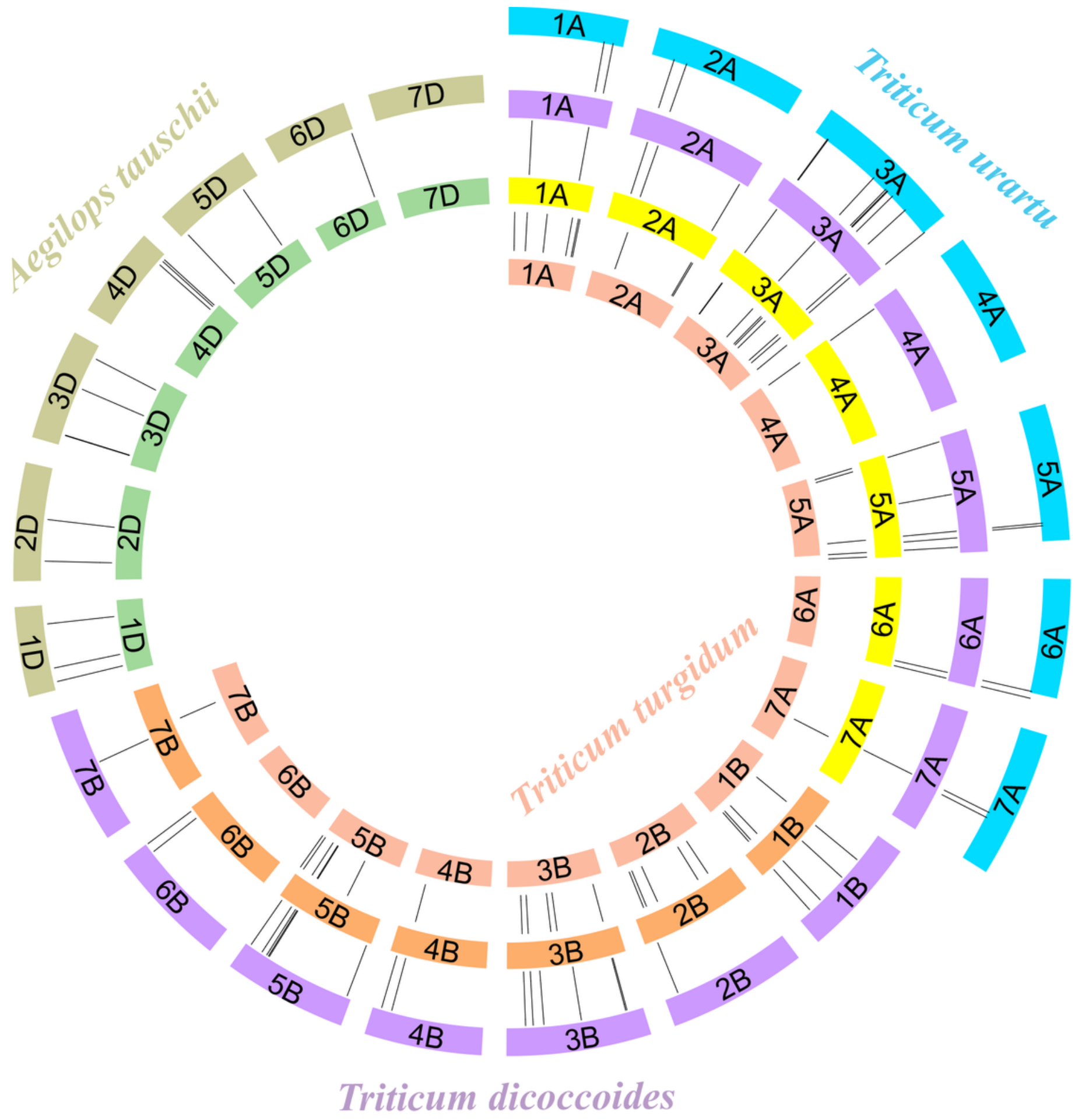

Figure 4

Syntenic analysis of IQD genes among common wheat and its progenitors. The genomes of Triticum urartu, Triticum dicoccoides, Triticum trugidum and Aegilops tauschii surround the central Triticum aestivum. Syntenic gene pairs are linked by lines. 
A

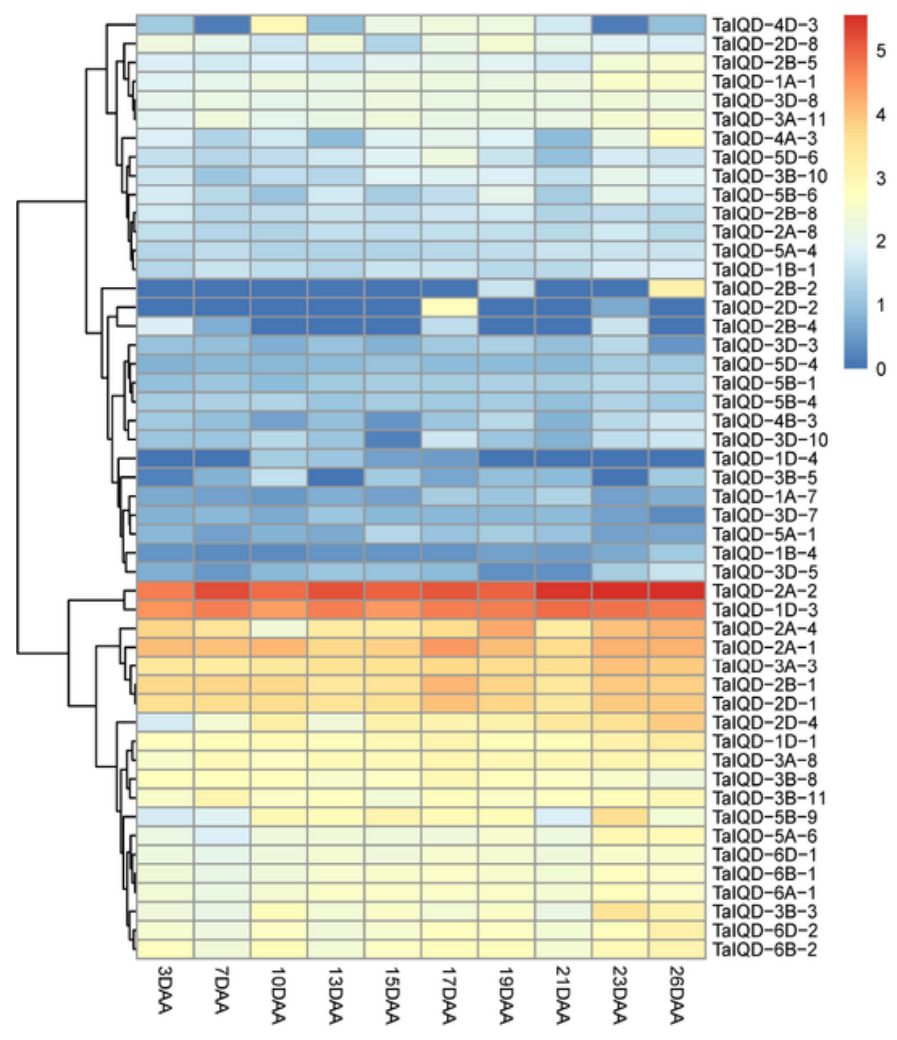

$\mathrm{B}$

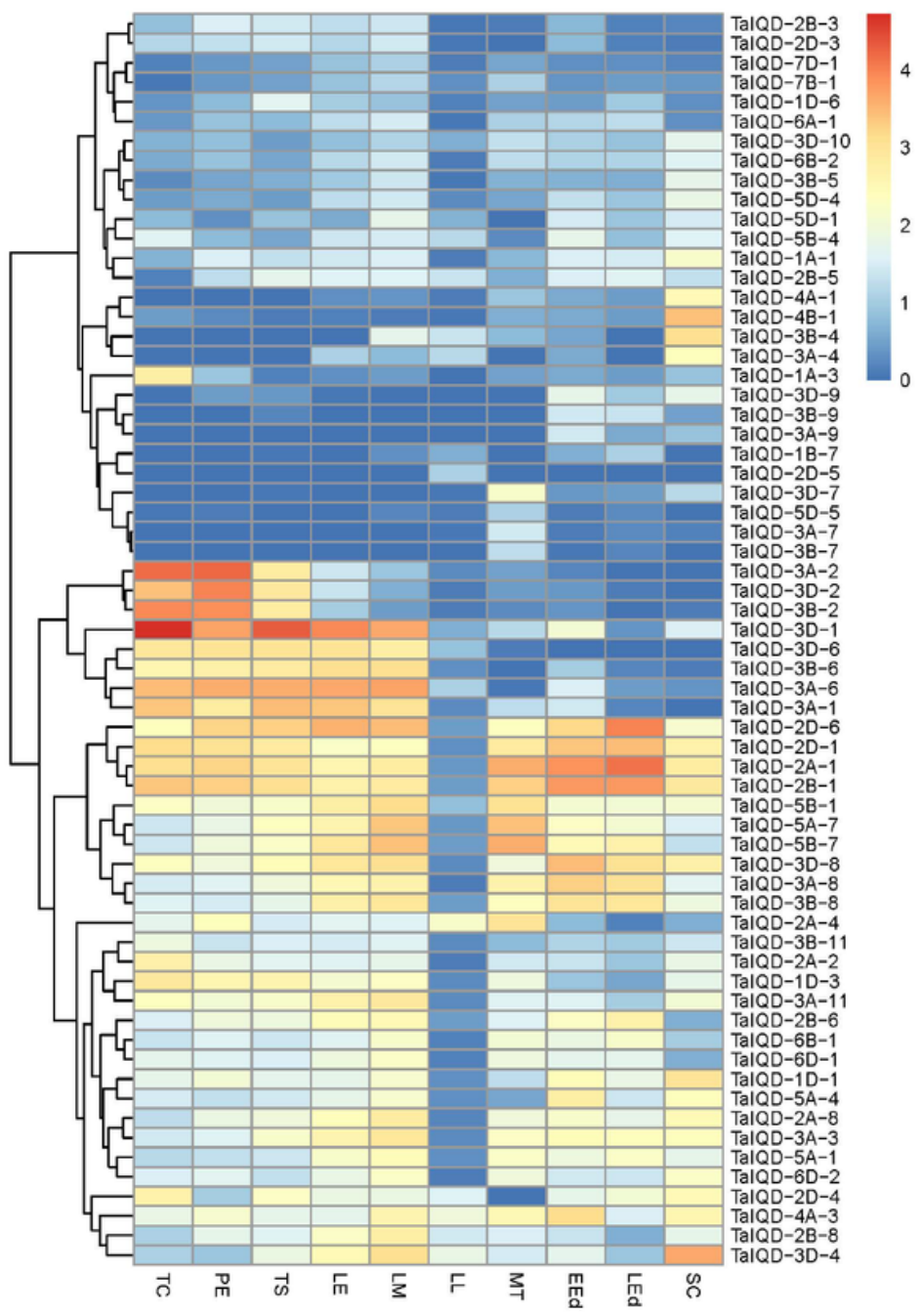

\section{Figure 5}

Expression pattern of TaIQDs during different developmental stage. Red indicates high expression and blue indicates low expression. The left represents gene clusters. A: 3 to 26 days after anthesis, B: ten different stages during embryo development. TC: two cells, PE: pre-embryo, TS: transition, LE: leaf early, LM: leaf middle, LL: leaf late, MT: mature, EEd: early endosperm, Led: late endosperm, SC: Pericarp. 


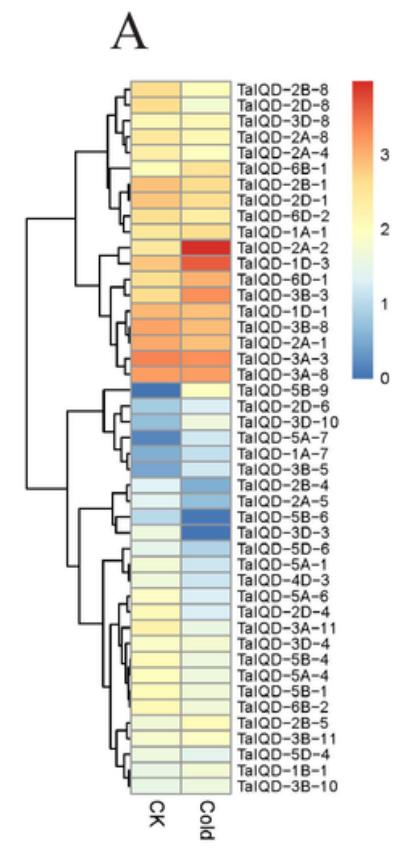

B

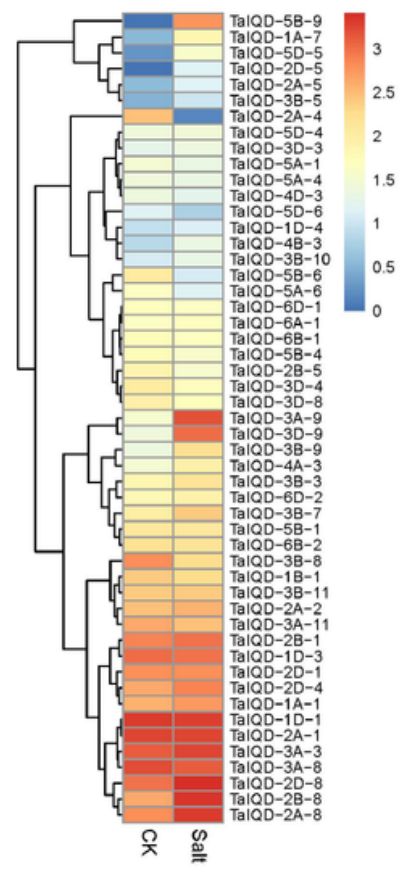

$\mathrm{C}$

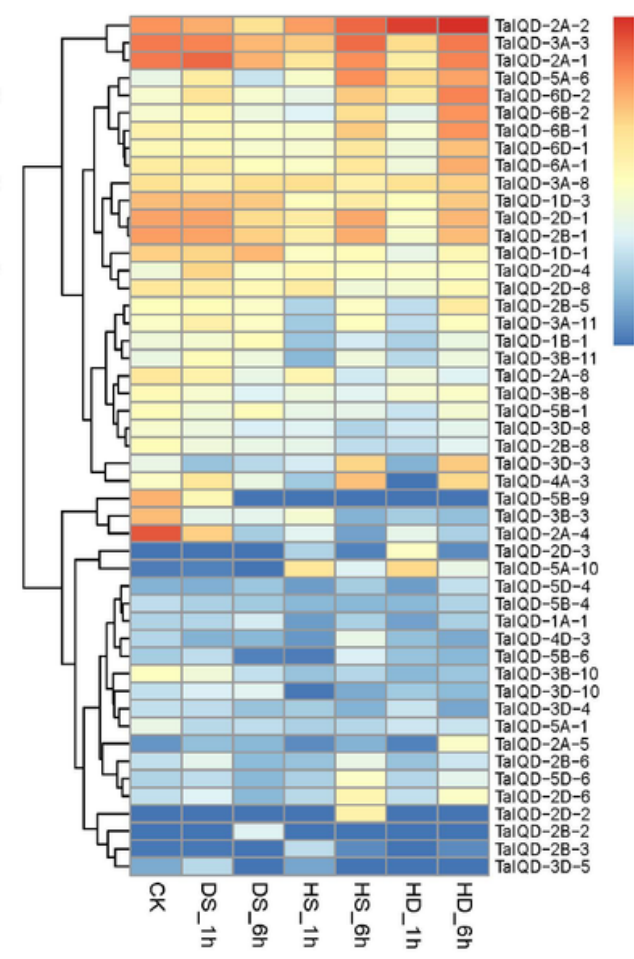

$\mathrm{D}$

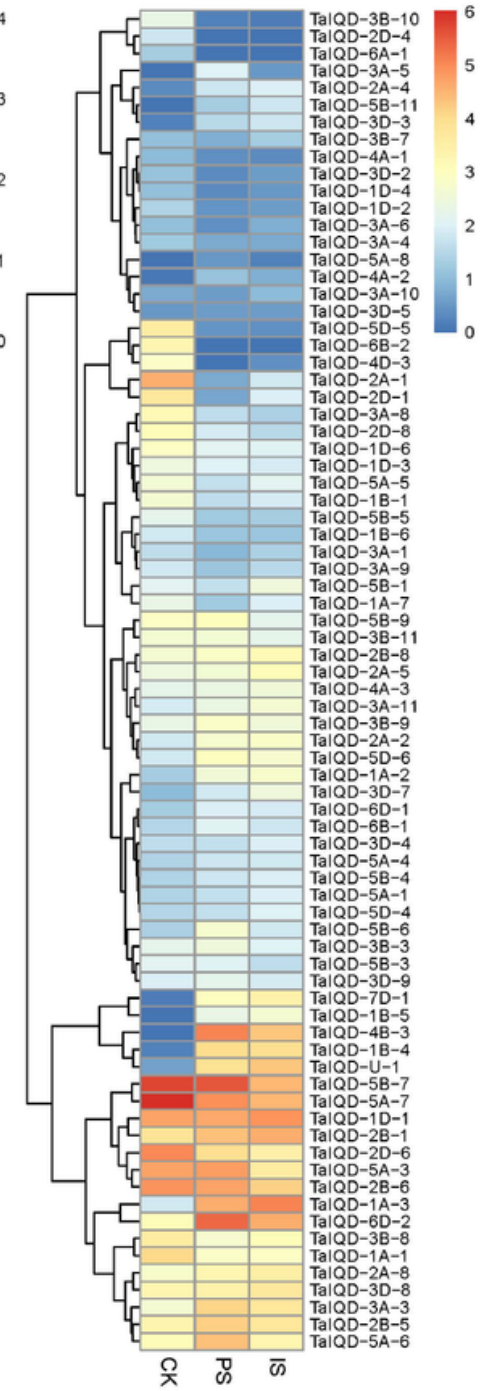

Figure 6

Expression analysis of TaIQDs in response to various abiotic stress. Red indicates high expression and blue indicates low expression. The left represents gene clusters. A: Cold, B: Salt, C: Heat with drought, D: Phosphorus and iron starvation. DS: drought stress, HS: heat stress, HD: heat with drought stress, PS: Phosphorus starvation, IS: Iron starvation. 
A

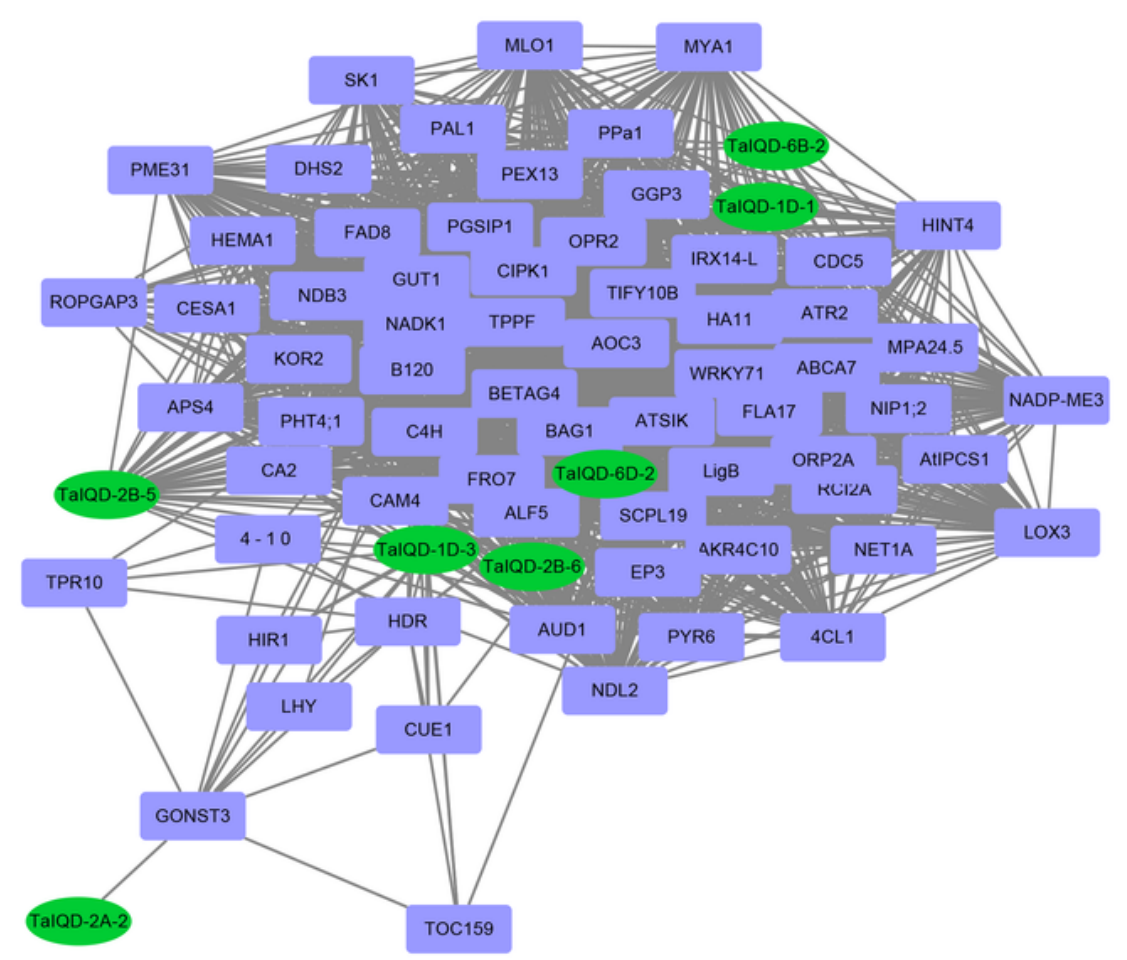

B

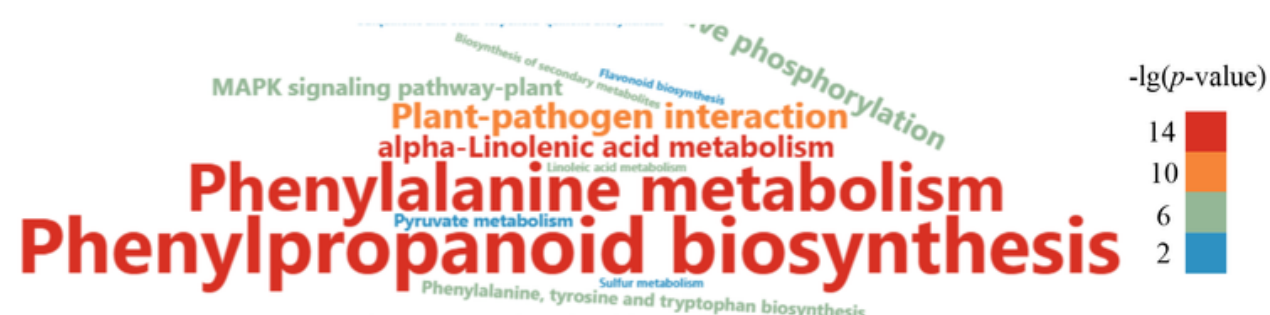

Amino sugar and nucleotide sugar metabolism
Carbon fixation in photosynthetic organisms

C

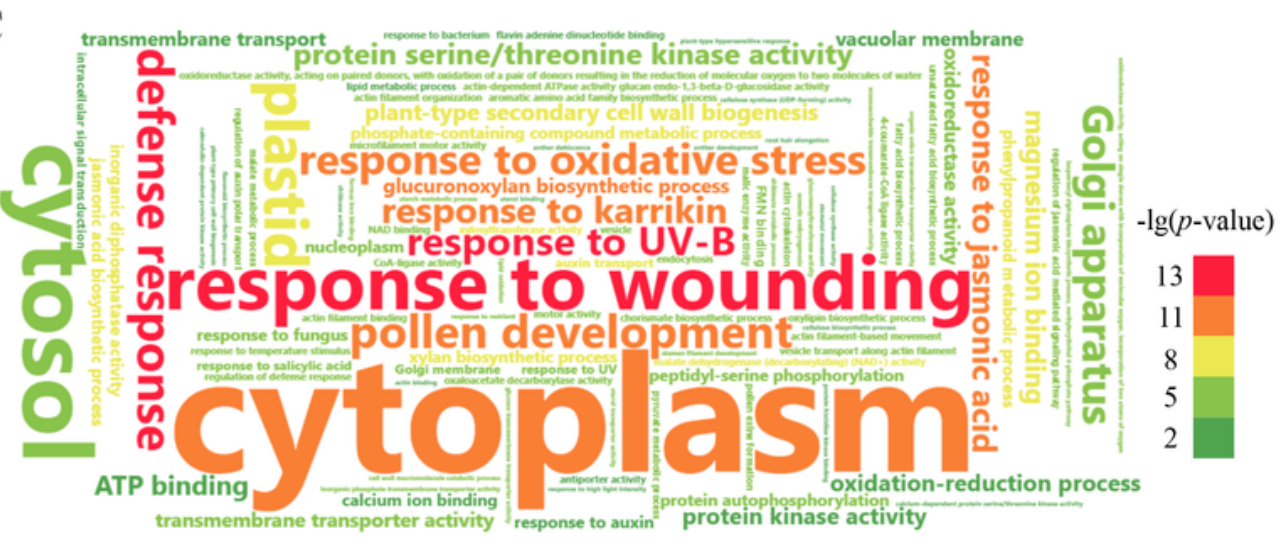

Figure 7

The co-expression network between TaIQDs and other wheat genes based on WGCNA analysis. A: WGCNA analysis, B: KEGG enrichment of TaIQD co-expressed genes, C: GO enrichment of TaIQD coexpressed genes. 

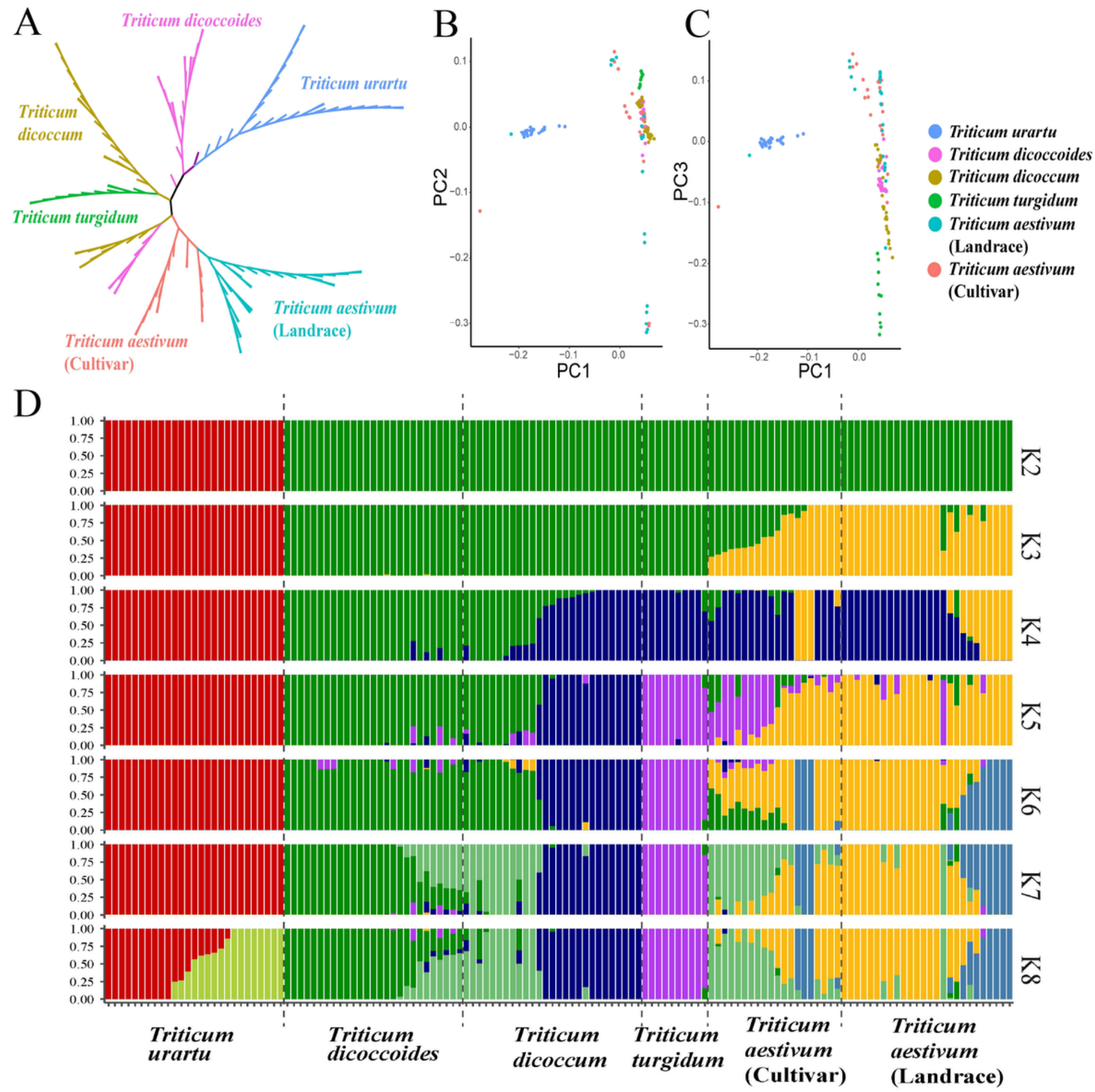

\section{Figure 8}

Phylogenetic relationships, PCA and population structure analysis for the group A genomes based on TaIQD-related SNPs. The SNPs from the A subgenome/genome of Triticum urartu, Triticum dicoccoides, Triticum dicoccum, Triticum turgidum and Triticum aestivum were used. A: Neighbor-joining phylogenetic tree, B: PCA analysis of PC1 vs PC2, C: PCA analysis of PC1 vs PC3, D: Population structure was estimated by ADMIXTURE with the $\mathrm{K}$ range from 2 to 8. 


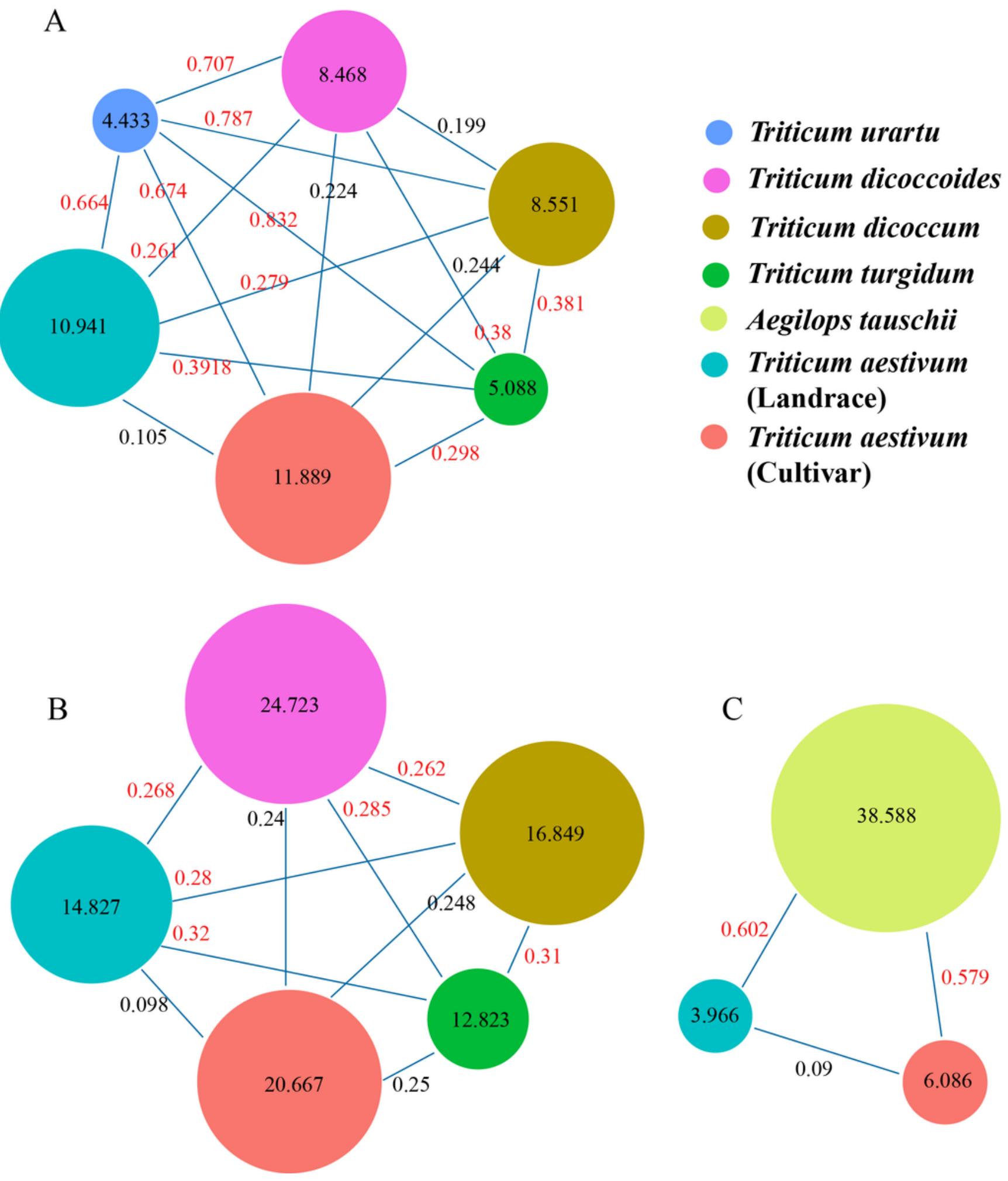

Figure 9

Distribution of nucleotide diversity $\left(\pi \times 10^{2}\right)$ and $F_{s t}$ values across the group A, B and D genomes, respectively. The $F_{s t}$ values larger than 0.25 are marked with red. A: A subgenome, B: B subgenome, C: D subgenome. 


\section{Supplementary Files}

This is a list of supplementary files associated with this preprint. Click to download.

- FigureS1.pdf

- Figures2.pdf

- Figures3.pdf

- FigureS4.pdf

- FigureS5.pdf

- FigureS6.pdf

- FigureS7.pdf

- Legendsforadditionalfiles.docx

- SupplementTables.xlsx 